1970 Membership Directory

1969-1970 NALLD MEMBERSHIP LIST

(Corrected to Feb. 1, 1970)

Permission for Commercial Use Must Be Obtained From

The NALLD Executive Secretary.

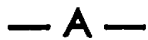

AASKOV, RUTH L., DLL, Augsburg Col., Minneapolis, Min. 55404

ACADEMIC LIB. HQTS-DLWIC, Bldg. No. 302, Presidio of Monterey, Monterey, Cal. 93940

ACQUISITIONS LIBRARIAN, N. Tex. State Univ., Lib. Dep., Denton, Tex. 76203. ADAM, RICHARD, DLL, Western Reserve Aca., Hudson, Oh. 44236.

ADAMS, KENNETH R., Weber State Col,, FLL, Ogden, Ut. 84403.

ADAMS, MRS. SUDIE M., Asst. Prof. Span. and Port., Baylor Univ., Box 159, Waco, TX. 76703.

ADCOCK, DWAYNE, Coord. FL, Eugene Pub. Schools, 200 N. Monroe St., Eugene, Or. 97402.

ADORNO, ROLENA K., Dir. Univ. of Hartford, 200 Bloomfield Ave., Hartford, Ct. 06617.

AGOSTINO, NELLIE, CHLD, East Hart High, 777 Burnside Ave., E. Hartford Ct. 06117.

AGUIRRE, A. A., Span. Dept. Hd., Ind. Univ. at South Bend, Northside Blvd., South Bend, In 46615 .

AGUIRRE, MRS. OLGA, Dir., Stratford Col., Danville, Va. 24541.

AHREN, MR. JOHN A., Westchester Coom. Col., 75 Grasslands Rd., Valhalla, N. Y. 10595.

AHRENS, MISS CHRISTA, FLLD, Ga. State Col., P. O. Box 415, Atlanta, Ga 30303.

AIKENS, H. F., DLL, Dalhonsie Univ., Halifax Nova Scotia, CANADA.

ALAMEDDINE, FAROUK, Sup. L.L., Wayne State Univ., 20023 Trinity Ave., Detroit, Mi. 48219.

ALBERTA, UNIV. OF, Romance Lang./Lang. Lab., Edmonton Alberta, CANADA Attn: Mrs. W. C. Deurloo.

AK. METHODIST UNIV., Foreign Language Dept., Box XL, Anchorage, Ak 99504. ALDRIDGE, REBECCA A., Instr. Mod. L, Northern Ok Col., 1008 E Oklahoma, Tonkawa, Ok 74653.

ALEMANY, GILBERTO R., Instr. in Span. Lang., Ricker Col., Houlton, Me 04730.

ALIMAN, LEE (LEON), Dir. FLL, San Francisco State Col., 1600 Holloway, San Francisco, Ca 94132.

ALIOTTI, VIRGINIA BROWN, Dir. of Lang. Lab., Madison Col., Box X, Harrisonburg, Va. 22801.

ALLEN, A. C., 4402 Fielding Rd., Wilmington, De 19802.

ALLEN, DR. HAL, Assoc. Prof. Ger., Hendrix Col., Conway, Ar. 72032.

ALLEN, ROBERT F., DLL, Rutgers Univ., 508 Newark Ave., Piscataway, N. Y. 08854

AMOR, Sr. Rose Teresa, Ch. Span. Dept., Molloy Catholic Coll., Rockville CTF, N. Y. 11570.

ALTMAN, LEE (LEON), Dir. F LL, San Francisco State Col., 1600 Holloway, San Francisco, Ca 94132.

AMBERT, GARY JOHN, DLL, E. Carolina Univ., Greenville, N. C. 27834.

ANNABEL, PATRICIA C., Chm., Slippery Rock State Col., Slippery Rock, Pa. 16057.

APPLEBAUM, MR. LEON, For. Lang. Dept., Seattle Central Comm. Col., 1415 Summit Ave., Seattle, Wa 98122.

AQUINAS, SISTER THOMAS, Span. Instr., St. Mary of Woods Col., St. Mary Woods, In 47876. 
ARANITI NAZMI, Dir. Lang., Quinnipiac Col., 303 Noble St., West Haven, Ct. 06516.

ARBAIZA, N. D., Chmn., Dept. For. Lang., Rockland Comm. Col., 145 College Rd., Suffern, N. Y. 10901.

ARENDT', J., Cnslr. in FL, Minneapolis Schools, 3709 48th Ave. So., Minneapolis, Mn 55406.

ARENSMAN, IMOGENE C., FL, Metropolis Comm. H S Dept., 1004 Catherine St. Metropolis, Il 62960.

ARC, SISTER JOAN OF, OP, Rosary High School, 8551 Greenfield Ave., Detroit, Mi 48228.

ARIEW, ROBERT, L. L. Dir., Univ. of Ill., 400 Briar Lane. Champaign, Il. 61820.

ARMAGNO, ANTHONY, 2000 Fifth Ave., River Grove, Il 60171.

ARMSTRONG, JOANNE, Assoc. Prof., Unive. of Tx. at El Paso, Box 5, El Paso, Tx 79902.

ARNOLD, KATHRYN, Dir. Lang. Lab., Adelphi Univ., Garden City N. Y. 11530.

ASSELIN, GUY, Supv., Lang. Lab., Carleton Univ., Ottawa 1 Ontario, CANADA.

ASSELT, JAN VAN, Head-Dept, of For. Langs., McPherson College, McPherson, Ks. 67460.

ATTIG, CECIL E., Supv., Ca. State Col., 800 N. State College Blvd., Fullerton, Ca 96231.

ATWOOD, MARGaReT M., Chmn. Mod. Lang., Husson Col., 1 College Cir., Bangor, Me. 04401.

AUCKLAND TEACHERS COLLEGE, The Librarian, 74 Epson Ave. Se. No. 3, Auckland, New Zealand.

AUCOIN, PROF. G. E., Box 760, Sydney Nova Scotia, CANADA.

AUGER, GILLES E., Nasson College, Springvale, Main 04083.

AUGUSTA, MARY SR, Prof. Fr. Span., Xavier Univ. of La., Pine and Palmetto Sts., New Orleans, La 70125.

AUSTIN, ROBERT P., LL Dir., Loyola Ac., 1100 N. Laramie, Wilmette, Il 60091.

BAAK, DR. L. E., Chmn., Dept. Mod. Lang., Morningside Col., Sioux City, Ia 51106.

BABIN, J. W., Assoc. Prof. of Fr., St. F. X. Univ., Antigonish NS, CANADA.

BAILLARGEON, THOMAS, Terryville High School, Terryville, Ct. 06786.

BAIMAS, JOHN C., Lang. Dept. Hd., Fitchburg, H. S., Fitchburg, Ma. 01420.

BAKER, ROBERT L., Chmn., Dept. of Rus., Middlebury Col., Middlebury, Vt. 05753.

BALBI, MARIO, Benedictine Mili School, 6502 Seawright Dr., Savannah, Ga. 31406.

BALCAEN, HUBERT, Lect., Univ. of Manitoba, 500 Dysart Rd., Winnepeg 19 Man CANADA.

BALL, MRS. JEANNIE, Chmn., Fr. Dept., Madeina School, Greenway, Va 22067.

BALTINS, MRS. VIVIJA N., P. O. Box 1012, Ft. Wayne, In 46801.

BALTIS, PAUL P., Lang. Lab. Supvr., 96-050 Farrington Hwy., Pearl, City, Hi 96782.

BANER, HUGo, Lindenwood Col., St. Charles, Mo. 63301.

BAPTIST UNIV. OK, Mod. Lang. Lab. Dir., Shawnee OK 74801.

BARGER, KENNETH S., Dir. LL, Columbia Comm. Unit 4, P. O. Box 1, Columbia, Il 62236 .

BARICEVIC, ELIZ M. Maryhurst Col., Maryhurst, Or 97036.

BAROLIN, MARTA, Westchester Comm. Col., Valhalla, N. Y. 12992.

BARR, CHARLES J., Dir. L.L., St. John S. Col., 5118 Seminary Rd., Camarillo, Ca 93010.

BARRETT, J. E., Hd. Lang. Dept., St. George S. School, W2929 Waikiki Rd.. Spokane, Wa. 99208.

BARRETT, ROBERT, Educ. Systems Consult., Lincoln Dr. and Johnson St., Philadelphia, $\mathrm{Pa} 19105$. 


\section{Membership Directory}

BARTH, REV. GILBERT, T O R Hd. Dept. of Mod. Langs., Col. of Steubenville. Steubenville, Oh 43952.

BASSETT, RALPH W., Supv. of FL, Portland Pub. Schools, 631 NE Clackamas St., Portland, Or 97208.

BATES, DR. MARGARET, Dir., Lang. Lab., 5914 Carlton Lane, Bethesda, Me. 20016.

BATTO, TERESA, Dir. Lang. Lab, Loyola Col., 4501 N. Charles St., Baltimore, Md 21210.

BAUDIN, PHILIP R., Coord. FL, Highlire Pub. Schools, P. O. Box 100, Seattle, Wa. 98166.

BAUMLIN, MLL, Milton Aca., 325 Randolph Ave., Milton, Ma. 02186.

BAXA, PAULINE, Dean, Dean Junior Col., Franklin, Ma. 02038.

BAXTER, MISS EVELYN N., Baldwin H. S., For. Lang. Coord., Pittsburgh, Pa. 15216.

BAYNARD, MRS. ANTHONY, Chd. FD, Maumee Valley Ctry. Day School, 1715 i. Reynolds Rd., Toledo, Oh 43614.

BEAUDOIN, HENRI, AV Dir., Cegep De Rouyn-Noranda, P. O. Box 1500, Rouyn P, Quebec, CANADA.

BECKER, REV. BRUNO, Mount Angel Semin., St. Benedict, Or 97373.

BEECHER, MRS. JEAN, Moravian Col., Bethelehem, Pa. 18018.

BEESON, SYLVIA, Hd. Mod. For. Lang., La Plata Sr. H. S., Oak Ave. LaPlata, La Plata, Md. 20646.

BEH, MISS YOLANDA C., Seamec Reg. Eng. Lang. Ctr., 104 Watten Estate, Singapore 11.

BELEW SOUND AND VISUAL, 1740 Edgemont Ave., Box 3002 SH, Bristol, Tn. 37620.

BELL, ELIZABETH, LL Dir., Allen Univ., Box 348, Columbia, S C 29204.

BELL, KEITH R., Prof. Mod. Lang., Mid-American Nazarene Col., 2030 College Way, Olathe, Ks 66061.

BENBOW, CAROLYN, Dir. LL, Tchrs. Coll, Columbia Univ., Box 66, 525 Wesi 120th. New York, N. Y. 10027.

BENITEZ, DR. MARIO A., Chmn, Mod. Lang. Dept., Texas A \& I Univ., Kingsville, Tx 78363.

BENOIST, GLORIA SR, LL Dir., St. Mary S Dominican Col., 7214 St. Charles Ave., New Orleans, La 70118.

BERGENS, PROF. ANDREE, Chmn. Dept. of Fr., Wilson Col., Chambersburg, Pa. 17701.

BERNADINE, M. SR., OSB, Thomas More Col., P. O. Box 85, Ft. Mitchell, Ky. 41017.

BERNIER, HENRI, Mod. Larg. Inst. and LIA, Walsh Col., 2020 Easton St. NW, Canton, Oh 44720.

BERNSTEIN, JAY, Lang. Advsr., Nassau Comm. Col., 79 Constable Lane, Levittown, N Y 11756.

BERNYNE, M. SR., FSPA-APOF, Viterbo Col., 815 South 9th St., Lacrosse, Wis. 54601.

BERTAUX, ALAN, Dir., 908 Windsor Rd., Baltimore, Md. 21208.

BIARNES, PIERRE, Sup. LL, Macomb County Comm. Col., 1450012 Mile Rd., Warren, Mi 48089.

BIBEAU, GILLES, CH Des Lab/DE Lang., Univ. De Montreal, C P 6128 (Faculte D Let) Montreal 3 Quebec. CANADA.

BISCHOFF, ELIZABETH M., Chmn. German Dept., Rand-Macon Women's Col., Lynchburg, Va. 24504.

BISSON, PAUL R., Dir. Lang. Lab., Greenfield Comm. Col., 124 Federal St., Greenfield, Ma. 01301.

BISHOFF, ELIZABETH M., C. G. D., Randolph.Macon Womens Col., R - M W C, Lynchburg, Va. 24503. 


\section{Membership Directory}

BIXBY, MRS. J. M., D L L, Juniata Col., Lang. Lab., Huntington, Pa. 16652.

BLACK, PROF. CARL, Asst. Prof. of Fr., Bard Col., Annandale, N. Y. 12504.

BLAIR, JOSEPH C., Dir. L.L., Lang. Lab. A-S, RM 3, Univ. of Missouri, Columbia. Mo 65201.

BLOSJO, R. V., Asst. Prof., Alderson-Broaddus Col., Philippi, W. V. 26416.

BLOUNT, JAMES P., Dir. Mod. Lang. Lab, Col. of William and Mary, Dept. of Mod. Lang., Williamshurg, Va. 23185.

BOARD OF EDUCA., Frechold Reg. High School Dist., 225 Schank Rd., Freehold, N: J. 07728.

BOBETSKY, PROF. V.. Dir. Lang. Lab., Polytechnic Inst. of Brook, 333 Jay St., Brooklyn, N Y 11201.

BODLANDER, HANS, Lang. Lab. Instr., U. of Bridgeport, Dana Hall, Bridgeport. Ct. 06602.

BODNAREK, VIRA, L L Dir., Chicago City Col., 3939 West 79th St., Chicago, Il. 60652 .

BOGART, WILLIAM F., DLL. Mansfield State Col., Blossburg, Pa. 16912.

BOHN, JERRY, Ast. Dir., Macalester Col., St. Paul, Mn 55104.

BOLDUC, MARCEL, Head F A D, Oblate Col, and Seminary, 149 Woodland St., Natick, Ma. 01760.

BOLDUC, PROF. R., Hd. Larg. Dept.. 251 Belmont St., Quinsigamond Com. Col., Worcester, Ma. 01605.

BOOT, DR. CHRISTINE. Asst. Prof., Drake Univ., 1422-25, Des Moines, Ia 50311.

BOOTH, EDWARD F., Conslt. of Mod. For. Langs., Dept. of Educa., Augusta, Me. 04330 .

BOOTHE, ROBERT O., Dir. of For. Langs., Ca. State Polytechnic Col., San Luis Obispo, Ca 93401.

BORN, WARREN C., Asst. Dir. MILA/ERIC, 62 Fifth Ave., New York N. Y. 10011. BOSTON UNIV., Dept. of Mod. Langs., 236 Bay State Rd., Boston, Ma. 02215.

BOSWAU, HERBERT. P. O. Box 98, Los Altos, Ca 94022.

BOUCHER, JOHN G., Lang. Lab. Dir., St. Anselm S. Col., Manchester, N. H. 03102.

BOUGARD, ROGER, Dir. Lang. Lab., North Carolina Col., 1406 1/2 Clarendon M., Durham, N. C. 27705.

BOUNDS, MRS. MURIEL, Teacher, Hermosa Junior High, 906 N. Butler, Farmington, N. M. 87401.

BOURAOUI, Fr. Lit. Dept., 232 Founders Col. York U70, 470C Keele St., Downsview Ontario, CANADA.

BOURASSA, FERNAND C. Head, Lord Beaverbrook HS FR. Dept., 111 Grafton Dr. SW, Calgary Alberta. CANADA.

BOURN, MRS. KATHERINE D., 262 Ferguson Rd., Manchester, Ct. 06040.

BOWEN, LOUISE H., Lang. Lab. Dir., Dartmouth Col., 201 Bartlett Hall, Hanover. $\mathrm{N} \mathrm{H} 03755$.

BOY, MRS. STELLA M., FL Coord., Watertown High School, Watertown, Ma. 02172 .

BOYDE, GEORGE E., L. L. Dir., Lafayette Col., 609 High St., Easton, Pa. 18042.

BOYDEN, PATRICK C.. Lang. Lab. Dir., Kent State Univ.. Kent, Oh 44240.

BRADLEY, PETER L., RFD 3, Quarry Rd., Middlebury, Vt. 05753.

BRADSHAW, M. E., 4431 Durer Pkwy., Sacramento, Ca. 95823.

BRADY, NORMA, Inter. American Univ.. Box 1293, Hato Rey, P. R. 00919.

BRATNOBER, H. L. JR., Chmn., For. Lang. Prog., SW Minnesota State Col., Marshall, Mn. 56258.

BRENDAN, ROSE SR. OP, Barry Col., 11300 NE Sec. Ave., Miami, Fl 33161.

BRENTON, MRS. V., Dir. LL Vincennes Univ. Jr. Col., Vincennes, In 47591.

BRETT, KENNETH C., Dept. of For. Lang., Univ. of Mt., Missoula, Mt 59801.

BREVARD COL., Dir. Lang. Lab., Brevard, N C 28712.

BREYMAN, MRS. CABYRON, Assoc. Prof. Chmn, Fr. Dept., Ca Lutheran Col., Thousand Oaks, Ca 91360. 


\section{Membership Directory}

BRIGGS, MRS. BECKY A., Mi. State Univ., LL a-126 Wells Hall, E. Lansing, Mi 48823.

BRIGOLA, DR. ALFRED, Coord. FL Lab. Dir., Univ. of Redlands, Redlands, Ca 92373.

BRINK, M. CAROLYN SR. RSM, Our Lady of Cinn. Col., Edgecliff-Walnut Hills, Cincinnati, Oh 45206.

BRITES, JAMES, FL Hd., The Nenley School, 86-86 Palo Alto St., Holliswood, N. Y. 11423.

BRITTAIN, MRS. F. S., W. Chicago Comm, H. S., 326 Joliet St., W. Chicago, Il 60185 .

BRODIN, DR. DOROTHY, Lehman Col., Bedford Park Blvd. W., Bronx, N. Y. 10468.

BROSSMAN, ERIKA S., Asst. Dir. ML Dept., Albright Col., Reading, Pa. 19604.

BROSSMAN, DR. LUTHER, Albright Col., Reading, Pa. 19604.

BROST', S. RAY. Asst. Prof. Ger., Lycoming Col., Williamsport, Pa. 17701.

BROWN, C. P., For. Lang. Dept., Western Ky. Univ., Bowling Green, Ky. 42101.

BROWN, ELDON I., L L Dir., Springfield Jr. Col., 1500 N. Fifth St., Springfield, Il 62702 .

BROWN, FLORENCE G., Ch. A S S, Defense Lang. Ins. Sup. Com., 5312 Salisbury, El Paso, Tx 79924.

BROWN. S. W., Prof. of Mod. Langs., Knox Col., Galesburg, Il 61401.

BRUGEYROUX. MARIE-LOUISE, French Specialist, 1220161 St., Edmonton 21 Alb. CANADA.

BRULL, EDGAR, R. I. Jr. Col., Mod. Lang. Dept., 199 Promenade St., Providence, R. I. 02908.

BRUNER, WILBUR J., William Jewell Col., Liberty, Mo 64068.

BSHARAH, M. ALBAN SR., Dir. Lang. Lab., Coll. of New Rochelle, New Rochelle. N. Y. 10801.

BUCK, DR. GEORGE C., Dir. Lang. Lab., Univ. of Wa., 118 Denny Hall, Seattle, Wa 98105 .

BUNDY, BONNIE, Dir. F L. The Defiance Col., L C Olds, Defiance, Oh. 43512.

BURR, YOLANDA, L. L. Supvr., Alfred Univ.. RD No. 3, Wellsville, N. Y. 14895.

BUSHALLOW, THOMAS, Supv. FL, Shaker HS, Latham, N. Y. 12110.

BUTEAU, MAGDELHAYNE, Chmn., The St. Joseph Tchrs. Col., 3465 Durocher St., Montreal Quebec, CANADA.

CAEFER, RAYMOND J., Hd. FLD, Wellesley Public Schools, Seaward Rd., Wellesley Hills, Ma. 02181 .

CAHILL, DANIEL P., Univ. of Conn., Storris, Ct. 06268.

CALLANAN, JAMES C., Dir. For. Lang. Lab., Southern Il. Univ., Edwardsville, Il 62025 .

CALVIN, THOMAS F., Dir. For. Lang., Marshfield Public Schools, Marshfield, Ma. 02050 .

CAMPA, DR. ROMAN DE LA, Chmn. Mod. For. Lang., Briar Cliff Col., Sioux City. Ia 51104.

CAMPBELL, MRS. G. M., Senior H. S., 160 Broad St., Bloomfield, N. J. 07003.

CANDIOTTI, JOSEPH, United Nations Dev. Prog., P. O. Box 24, Magadiscio Somalia E AF.

CAPRETZ, PIERRE J., Dir. Lang. Lab., Yale Univ., 111 Grove St., New Haven, Ct. 06510

CARR, EMORY W., Instr.. Marshall Univ., P. O. Box 114, Huntington, W. V. 25701.

CARTIER, F. A., Dir. of DEV., Defense Lang. Inst. Eng., 7415 Buckskin Lang. Sch., San Antonio, Tx 78227.

CARTWRIGHT, RICHARD, Chmn., Mod. Lang. Dept., Mt. Hermon School, Mt. Hermon, Ma. 01354. 


\section{Membership Directory}

CARULLO, MRS. MARIA E., 61 White Oak St., New Rochelle, N. Y. 10801.

CATHCART, KENT, Dir. LL, Univ. of S. Ca., English Communication, Los Angeles, Ca. 90007.

CERVIZZI, CHRISTINE, C F L, Northbridge High School, Linwood Ave., Whitinsville, Ma. 01588.

CHANDLER. DAVID G., St. Olaf Col., Northfield, Mn. 55057.

CHANG, GERALD K. J., 4457 Puu Panini Ave., Honolulu, Hi 96816.

CHATAGNIER, LOUIS J., 4874 Cote Des Neiges, Apt. 1401, Montreal PQ, CANARA.

CHERRY, C. MAURICE, Dir. Mod. Lang. Lab., Furman Univ., Greenville, S. C. 29613.

CHERRY, RALPH, Instr. and Lang. Lab. Dir., Southeastern State Col., Durant, Ok 74701 .

CHILDS, LORA E., Lang. Lab. Dir., Wilson High School, 1151 SW Vermont St., Portland, Or 97219.

CHIVERS, JOHN, Chmn., German Dept., Phillips Aca., Andover, Ma. 01810.

CHOMAR. DR. ORLANDO, Sp. Instr., Col. of Mt. St. Jos. on Ohio, 5701 Delhi Rd., Mt. St. Joseph, Oh 45051.

CHOMEI, TOSHIKO, Instr., Ochanomizu Univ., Otsuka 2 Chome, Bunkyo-Ku, Tokyo, Japan.

CHRISTMAN, HENRY R., L L Dir., Kutztown State Col., 215 Wellington Ave., Reading, Pa. 19609.

CICERO. A. E., Prof., Allegheny Comm. Col., 809 Ridge Ave., Pittsburgh, Pa. 15212.

CIFFORD, NATHANIEL H., Villa "Les Ciogognes", Quartier Pierredon, 13 Eguilles, FRANCE

CIRUTI, MISS JOAN E., Dept. of Spanish Chmn., Mt. Holyoke College, So. Hadley, Ma. 01075

CLAESGES, DR. A. W.. Dept. of For. Lang. and Lit., So. Methodist Univ., Dallas, Tx. 75222.

CLARK, BEN T., Cord. of Lang. Studies, Univ. of Ca., Merrill Col. UCSC, Santa Cruz, Ca. 95060

CLARK. CLYDE L.. Assoc. Prof.. Span.. Butler Lniv.. Indianapolis. In. 46208

CLARK, EDWARD F.. Asst. Prof.. Dept. of For. Langs., Moorhead State Col.. Moorhead, Mn.. 56560

CLAUDEL. CALVIN A.. Box 622. Salisbury State Col.. Salisbury, Md. 21801

CLAY, GERALD. 108 Berkeley Dr.. Florence. Ky. 41042

COBB. MRS. J. V.. 209 Black Bluff Rd.. Rome. Ga. 30161

COFFIN. MRS. T. C.. LL Asst.. The Baldwin School. Bryn Mawr. Pa. 19010

COLECCHIA. DR. FRANCESCA. Dir. Lang. Lab. Duquesre Univ., Pittsburgh, Pa. 15219

COLL. PAUline, Chmn.. For. Langs.. Averett Sol.. 135 Mulberry Rd., Danville, Va. 24541

COLLINS, RALPH S.. Chmn., Dept. of For. Lang., Maryland Col., 1741 Linda Ln., Maryville. Tn. 37801

COLOMBO, RUTH W., Lang. Dept.. Natick H. S.. 15 W. St.. Natick. Ma 01760

COMM. HS DIST. NO. 88. 1250 S. Ardmore Ave.. Villa Park. Il. 60181

COMMISSION SOLAIRE REG. LALONDE, Case Postale 3500, La Sarre, Quebec, CANADA

CONKEY. MRS. JEAN S., Supv. Lang. Lab., Wheaton Col., Norton, Ma. 02766

UNIV. OF CT.. Dir. Larg. Lab., Storrs, Ct. 06268

CONNELL, MR. THOMAS F. O., Admin. of Lang. Lab., Smith Coll-Wright Hall, Northampton, Ma. 01060

CONNELLY, MR. CYRIL, Summit Junior High School, 272 Morris Avenue, Summit, N. J. 07901

COOK, MARGARET A.. LL Dir., Ind. Univ. at Inpolis, 222 E. Michigan St., Indianapolis, Ind. 46204 


\section{Membership Directory}

COON, REV. DAVID P., A Hdmster., Iolani School, 563 Kamoku St., Honolulu, Hi. 96814

COOPER, C. T., Asst. Prof. of Span., Lebanon Valley Co., Annville, Pa. 17003

COOPER, DR. D. CHAVY, Chmn. Dept. Mod. Lang., Keuka Col., Keuka Park, N. Y. 14478

COOPER, HENRY F., Dir. Lang. Lab., Univ. of Or., Romance Lang. Dir., Eugene, Or. 97403

CONWELL, DR. MARILYN J., DLL, Rosemont Col., 251 W. Dekalb Pike, King of Prussa, Pa. 19406

COSTA, RONALD KENT, Dir. Lang. Lab., Mass. St. Col., Framingham, Ma. 01701

COTE, PAUL E., Dir. Lang. Lab., Avon High School, Avon, Ct. 06001

COROLVILLE CENT. EL. SCH., Central Processing, 501 6th St., Coralville, Ia. 52240

CORPACI, JOHN, Ch. Lang. Dept., Cheshire Aca., Box 306, Cheshire, Ct. 06410

CORRY BRO. H. MICHAEL, Fsc. Lang. Lab Dir., Christian Bro. Aca., Lincroft, N. J., 07738

CORTES, ELADIO, Lang. Lab. Dir. and Span. Dept. Chmn., Rutgers Univ., Camden, N. J-. 08102

COSMAN, MRS. T., Dir. Lang. Lab., Barnard Col., 11 Lehman, 26 Claremont Ave./ Hall, New York, N. Y. 10027

COTE, NORMAN R., Head F L D, North Shore Comm. Col., 3 Essex St., Beverly, Ma. 01915

CORTEZ, DAVID L., Span. Tea., Clearfield High School, Clearfield, Ut. 84015

COUEY, EARL F., LL Dir., Sonoma State Col., 1801 E. Cotati Ave., Rohnert Park, Ca. 94928

COURNOYER, MADELEINE A., Ch. FL ,Provios East High School, First Ave. and Madison, Maywood, Il. 60153

COURTOIS, NELLY, Newton Col. of Sacred Heart, 885 Centre St., Newton, Ma. 02159

COX, SHIRLEY O., L. L. Dir., Auburn Unive., FL Dept., Auburn, Al. 38630

COYNE, MARTIN J. Tech. Asst. Lang. Lab., U. of Ma-Boston, 100 Arlington St., Boston, Ma. 02116

CRAIG, MRS RUTH PARLE, Dir Lang. Lab. and Chmn. Lang. Dept., Santa Rosa Junior Col., Santa Rosa, Ca. 95401

CRAIGHILL, KARY NUTE, 4533 38th St. So., St. Petersburg, Fl. 33711

CREAMER, JOHN F., D. L. L., Hudson H. S., Cottage St., Hudson, Ma. 01749

CRIMINALE, LEONARD R., Elmira Col., Elmira, N. Y. 14905

CROVER, M. ANSELM SR., Dir. LL, Col. of the Holy Names, 3500 Mountain Blvd., Oakland, Ca. 94619

CROWNER, DR. DAVID, Lang. Lab. Dir., Gettysburg Col., Gettysburg, Pa. 17325

CRUVELLIER, J.P A., Head Mod. Lang. Dept., Lower Canada Col., 4090 Royal Ave., Montreal 261, CANADA

CRUZ, CARLOS M., Supv. Lang. Lab., Univ. of Rochester, River Campus Sta., Rochester, N. Y. 04627

CUNEO, WILLIAM J., Dire. Lang. Lab., Holyoke Commu. Col., 170 Sargeant St., Holyoke, Ma. 01040

CUNNINGHAM, M. J. JR., Ch. LD, New Mexico Junior Col., Lovington Hwy, Hobbs, N. M. 88240

CURRIE, MRS. EVA G., Dir. Spch. Lab., Univ. of Tx., Dept. of Spch., Austin, Tx. 78712

DACEY, D. P., KH 316, State U. Col. At Buffalo, 1300 Elmwood Ave., Buffalo, N. Y. 14222

DAIDAKIS, JOYCE, Dir. L L , Johns Hopkins Univ., Lang. Lab., Baltimore, Md. 21218 
DAILEY, BRO. ED, Dept. Chmn., St. Edward High School, 13500 Detroit Ave., Lakewood, Oh. 44107

DALGO, RODNEY C., Dir LL, Univ. of S C, Lang. Lab., Columbus, S. C. 29208

DARCEY, JOHN M., FL Supvfl, 7 Whiting Lane, W. Hartford, Ct. 06119

DARRAS, MICHAEL, Assoc. Prof. of Fr., Mem. Univ. of Newfoundland, St. John S. NFL, CANADA

DAY, PHILIP STEPHEN, Asst. Prof. Fr., Queen S. Univ., Kingston, Ontario, CANADA

DE BONE, DR. G., Head of Dept., La Grange Col., La Grange, Ga. 30240

DECATOLI, MISS DOLORES, Lasalle-Peru Twp. High School, 541 Chartres St., La Salle, Il. 61301

DECOCK, MRS. THERESA, R R 1, Box 126, Cottonwood, Mn. 56229

DELANEY, JOHN, Wolcott High School, Wolcott, Ct. 06716

DELANOEYE, MRS. JOHN, H FL L, Ca. State Col., 5151 State Col. Dr., Los Angeles, Ca. 90032

DENNIS, LARRY KENT, Dir. Instructional Media, Concordia Col., Moorhead. Mn. 56560

DEONES, JIMMIE, Asst. Prof., Olney Central Col., Fr. Dept., OIney, Il. 62450

DE PAUL, SR. MARIE, Ch. Lang. Dept., Bishop Gallagher, 19360 Harper Ave., Harper Woods, Mi. 48236

DE PEAUZ, REV. R. C., Chmn., Dept. of Mod. For. Lang., St. Norbert College, W. De Pere, Wis. 54178.

DERMINER, MISS NANCY, Asst. Prof. of Fr., Limestone Col., Gaffney, S. C. 29340

DEROUEN, DR. LARRY, Dept. of Lang., McNeese State Col., Lake Charles, La. 70601

DE SALVO, BENNIE T., Hall High, 50 S. Main St., W. Hartford, Ct. 06051

DESROCHERS, LEGER, 94 De L Acadie, Sept-Iles, Quebec, CANADA

DETI, DANIEL, Chmn. Lang. Dept., Col. of the Desert, Palm Desert, Ca. 92260

DE TORRE, EMILIO E., Queens Col. of the City of N. Y., Flushing, N. Y. 11367

DEVARGAS, MR. DIEGO, FL Coord., Carlsbad Municipal School, 103 W. Hagerman St., Carlsbad, N. M. 88220.

DEVINE, REV. F. J., SJ, Chmn. Dept. Fr., St. Mary S. Univ., Halifax, N. S. CANADA

DEVLIN, JUSTINE M. Head-MFL Dept,, Lawrence High School, Lawrence, Ma. 01841

DICICCO, MISS ELAINE, Cmn. F. L. Dept., Concord-Carlisle High, Concord, Ma. 01742

DICKERMAN, WILLIAM C., Dir. LL, Univ. of Houston, 3801 Cullen Blvd., Houston, Tx. 77004

DIETRICH, ELMER, Hd. of Lang. Dept., High School, Miller, S. D. 57362

DIMPEL, DORIS E., 756 Eagle St., Dunkirk, N. Y. 14048

DIRLAM. PROF. JANET, Box 6093, SFA Sta., Nacogdoches, Tx. 75961

DIVINCENZO, PROF. VITA, Dir. L L, Villanova Univ., Spring and Hilldale Rd., Malvern, $\mathrm{Pa} .19354$

DIXON, EDWARD, Chmn. Eng. Dept., Se. Campus Chi. City Col. 8600 S. Anthony Ave., Chicago, Il. 60617

DIXON, FRANCE P., Lang Lab. Dir., McKendree Col., Lebanon, Il. 62254

DODDY, MICHAEL, S., Tch. Span., No. Bergen High School, 190 West 168th St., Bronx, N. Y. 10452

DOHERTY, J. C., Hd. Lang. Lab., St. Malachy S., H. S., Leinster, St., St. John, N. B., CANADA

DONATELLI, JOSEPH, L L Dir., Instr. of Span., Univ. of Akron, Akron, Oh. 44304

DORNEMANN, WILLIAM E., Lang. Lab. Dir., Dickinson Col., Carlisle, Pa. 17013

DOVER, HUGH C., Mgr, and Adir/11, Duke Univ., 508 N. Buchanan Blvd., Durham, N. C. 27701

DOWLING, CHARLES, Dir. Lang. Lab., Baldwin-Wallace Col., Berea, Oh. 44017 
DOWNING, MRS. B., West Tx. State Univ., Canyon, Tt. 79015

DRAGONE, DR. O., Dir. M L S, A I C, State St., Springfield, Ma. 01109

DRANEY, MR. EDWARD, Chester Electronic Labs., 17 Navesink Dr., Monmouth Beach, N. J. 06412

DUKAS, DR. VYTAS, Dir. For. L. L., San Diego State Col., 5402 Col. Ave., San Diego, Ca. 92115

DRUMMOND, DONALD L., Audio Coord., San Antonio Col,, 1001 Howard St., San Antonio, Tx. 78212

DUCRETET, PIERRE R., Asst. Dir., Prof. and Asst. L. L. Dir., Univ. College 213, U. of Toronto, Toronto 5, Ontario, CANADA

DUNAWAY, AGNES, Ch. FL, Riverside High School, 1615 E. Locust St., Milwaukee, Wi. 53211

DUNBAR, DR. H. B., Ch. Div. of Lib. Arts, NYC Comm. Col., 300 Jay St., Brooklyn, N. Y. 11201

DURCHARME, SISTER M. J., Chmn. Mod. Lang., Rivier Col., Nashua, N. H. 030ล0

DURDEN, JOHN D., 4738 Everhart Dr., North Canton, Oh. 44720

DURHAM, BILL. 201 Strode Tower, Clemson Univ., Clemson, S. C. 29631

DUVAL, F. A., Chmn. Clas./Mod. Lang., Cornell Col., 710 8th Ave. N., Mt. Vernon. Ia. 52314

DVORAK, CAROL SR., Lang. Lab. Dir., Mt. Mercy Col., 1330 Elmhurst Dr. NE. Cedar Rapids, Ia. 52402

\section{$-E-$}

EASTMAN, V. DAVID, Lab. Dir., E L S Lang. Ctr. I, A, 511 S. Bonnie Brae, Los Angeles, $\mathrm{Ca} .90057$

ECHOLS, SAM G., Dir. Lang. Lab., Salem State Col., 4 Brown St., Salem, Ma. 10970

ECONOMOS, R. M., Prof., Pace Col., 41 Park Row, New York, N. Y. 10038

EDIE, MRS. JOHN, Ch. Fr. Dept., Northrop Collegiate Sch., 511 Kenwood Pkwy., Minneapolis, Mn. 55403

EDLINE, CLAUDE, L. L. Dir., Gilman School Inc., 5407 Roland Ave., Baltimore, Md. 21210

EDMONDS, WILLIAM B., Chmn., Mod. Lang., Gainesville Jr. Col., Gainesville, Ga. 30501

EDWARDS, DAVID WAYNE, DLL, Emory Univ., Dept. of Romance Langs., Atlanta, Ga. 30322

EHRAT, Ken, Lang. Lab. Dir., The Lanier Co., 111 W. Belmont, Pensacola, Fl. 32501

EILERTSON, E. J., Prof. of Ger., Carroll Col., 414 W. Park Ave., Waukesha, Wis. 53186

EISENSTADT, RALPH A., LLD, West Chester State Col., For. Lang. Dept., West Chester, Pa. 19380

ELDON, MRS. JANE R., Dir. Lang. Lab., N. Central Col., Naperville, Il. 60540

ELGUEZABAL, D., Dir. L L , Loyola Univ., P. O. Box 81, New Orleans, La. 70118

ELIFSON, MRS. LUCY, Waukesha High School, 400 N. Grand Ave., Waukesha. Wi 53186

ELLIS, BARRY L., 14804 Beech, Daly Rd., Detroit, Mi. 48239

ELLIS, RAY, Prof., Hardin-Simmons Univ., Abilene, Tx. 79601

ELMENDORF, WILLIAM E., Dir. Lang. Lab., Western Wa. State Col., Bellingham, Wa. 98225

ELMORE, ROGER J., AHMFL, Cumberland Col., Box 121 Cumberland Col. St., Williamsburg, Ky. 40769

ELTON, MAURICE G., Southern Methodist Univ., Clements Hall, Dallas, Tx. 75222.

EMERY, EDMOND L., Chmn., Lang. Dept., Milford Aca., 150 Guld St., Milford, Ct. 06460 
EMILIENNE, SISTER THERESF, Dir. Lang. Lab., Annhurst Col., Woodstock. Ct. 06281

EMILY, MARY SR., Fr. Inst./LL Dir., 1330 Elmhurst Dr. NE., Cedar Rapids, Ia. 52402

EMOND, NORMAN R., FL Dept./Chmn., Woodstosk Cca., Academy Rd., Woodstock, Ct. 06281

EPPINK, ALICE J., Lib., Center Applied Ling., 1717 Mass. Ave. NW., Washington, D. C. 20036

ERICKSON, MRS. EVELYN, Box 104, Mahopar, N. Y. 10541.

ESPINOSA, LORENZO. Lang. Lab. Supv., Univ. of Ca.. Riverside, Ca. 92502

ESSA, DR. ROBERT, Chmn. FL Dept., Sierra Col., 5000 Rocklin Rd., Rocklin, Ca. 95677

EVANS, JAMES L., Tech., Supv., Univ. of Hawaii, P. O. Box 1698, Honolulu, Hi. 96896

EWALD, WILLIAM M., Dir. L. L., Concordia Col., Box 9-C. River Forest, Il. 60305

EWING, R. R. Jr., Dir. Lang. Lab., Washington and Lee Univ., Dupont 102, Lex. ington, Va. 24450

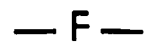

FAILEY, MRS. H., 540 Jones Ave., Toronto 6, Ontario, CANADA

FAIRLEY, MRS. HOPE, Dir. Lang. Lab., 51 Terraulay St., (Bay Camp), George Brown Col., Toronto 102, Ontario, CANADA

FAIRLEY, MAMIE, Hd. FL, Magnolia High School, Box 428, Moss Point, Mis. 39563

FANG, ILSE M., Prof. Suffolk Univ., 41 Temple St., Boston, Ma. 02114

FANTINI, ALVINO E., Dir. For. Lang., Experiment in Intnl. Liv., Kipling Rd., Brattleboro, Vt. 05301

FARRELL, BR., CFX, Chmn., Mod. Lang., Ryken H. S., Leonardtown. Md. 20650

FAULKNER, JAMES C., Chmn. For. Lang., State Univ. Col., 17 Cedar Ridge Rd., New Pailtz, N. Y. 12561

FAYE, MARIO R., Chmn. For. Lang. Dept., Tacoma Community Col., Tacoma, Wa. 98465

FECTEAU, LEO J., Head For. Lang. Dept., Barrington Public Schools, Barrington High School, Barrington, R. I. 02806

FEINBERG, ROSA CASTRO, Robert E. Lee Jr. High, $3100 \mathrm{NW}$ th Ave., Miami, Fl. 33127

FELSHER. DR. WILLIAM, Hd. Dept. For. Lang., Univ. of Evansville, Evansville, In. 47704

HIGH SCHOOL RESEARCH CTR., Felsooktatasi Pedagogiai, Kutatokozpont Budapest, VIII Rigo Utca 16 Hungary

FENNER, DR. REST JR., Hd. Lang. Dept., 91 South Orange Ave., Livingston. N. J. 07039

FERGUSON, LOU. Chmn. FL Dept., Glenbard East H. S., 1014 S. Main St., Lombard. Il. 60418

FERLAND. MARCIEN. Dir. of Lab., Univ. of Winnipeg. Winnipeg Man., CANADA

FERNANDEZ. DR. AUGUSTINE, Bishop Coll/A P R L, 3837 Simpson Stuart, Dallas. Tx. 75241

FERNANDEZ. DR. JOSE C.. 6 Lakeside Court, Plattsburgh, N. Y. 12901

FERSEN, NINA, Director. Lang. Lab., The Karl E. Weston Lang. Ctr., Williams Col., Williamstown. Ma. 01267

FICARRA. A. V., Asst. Prof., Western Conn. State Col., 181 White St., Danbury, Ct. 06810

FIELD. DOUGLAS A., Dept. of For. Lang., Maui Comm. Col., Kahului Maui, Hi. 96732

FINK, BRUCE L.. Lab. Dir.. Pasadena City Col.. 296 Ohio St., Pasadena, Ca. 91106

FINK, KARL T., Instr., Luther Col., 811 Iowa Ave., Decorah, Ia. 52101 


\section{Membership Directory}

FINN, BARTHOLOMEW J., Lang. Lab. Dir., 220 Golden Gate, San Francisco, Ca. 94102

FISHER, RONALD D., LL Dir., La Salle Col., 20th St. at Olney Ave., Philadelphia, Pa. 19141

FISK UNIV., Lang. Lab. Dir., Box 19, Nashville, Tn. 37203

FITCH, C. BRUCE, Dir. L. L., Transylvania Col., 300 N. Broadway, Lexington, Ky. 40508

FITZGERALD, BRO. AUGUSTINE, F S C, 200 Clinton Ave., Staten Island, N. Y. 10301

FLEETWOOD FURN CO. INC., Zeeland, Mi. 49464

FLEURANT, R., Pine Crest School, 1501 NE 62nd St., Ft. Lauderdale, Fl. 33308

FLITCROFT, NATALIE C., Emerson Jr. Sr. HS, Main St., Emerson, N. J. 07630

EDUC. RESOURCES DEPT. ADMIN., Univ. of South Fl., 4202 Flower Ave., Tampa, Fl. 33612

FORD, ANTHONY, Dir. Lang. Lab., Northeastern Univ., Boston, Ma. 02115

FORD, JOHN ELDON, Instr. Fr., Northland Col., 213 and Street E, Ashland, Wi. 54806

FORTNER, DEBORAH, Lan. Dir., Citrus High School, Inverness, Fl. 32650

FOURNET, JAMES F., Dir. LL, Southeastern La Col., 1004 S. Oak St., Hammond, La 70401

FOURNIER, ROBERT, Consult. Fl, St. Dept. of Educ., 17 Webster St., Suncook, N. H. 03275

FOWLER, BETTY, Conslt. For. Lang., Dept. Ed-Stanislaus City SC, P. O. Box 1697 Modesto, Ca. 95354

FOWLER, MRS. JULIA, FL Coor., Radnor Twp. Schools, Adm. Bldg., S. Wayne Ave., Wayne, $\mathrm{Pa} .19087$

FRANCISCO, DAVID, Dir., Dial Access, Forest Pk. Col., 5600 Oalkand, St. Louis, Mo. 63110

FRANKS, ANITA W., Span., Thayer Aca., 745 Washington St., Braintree, Ma. 02185

FRANKS, GENE, Dept. of For. Lang., No. Texas State Univ., Denton, Tx. 76203

FRAYNE, EVA, L L Coord., Parkland Col., 2 Main St., Champaign, Il. 61820

FRAZIER, ESTELLE C., Fr. Tchr., Academy Heights High School, P. O. Box 1210, Pinehurst, N. C. 28374

FRECHETTE, ERNEST A., Dept. For. Lang., Fl. State Univ., 1418 W. Call St., Tallahassee, Fl. 32306

FREEMAN, DOROTHY S., Inst. Fr., Paine Col., 404 Sheffield Cir., Augusta, Ga. 30904

FRENCH, BRUCE A., Dir. LL, New Haven Col., 300 Orange Ave., West Haven, Ct. 06516

FRENCH, JOANNE D., 550 Kelly Way, Palo Alto, Ca. 94306

FREVAL, DANIEL, Lab. Dir., Dickinson Col., Dept. Mod. Lang., Carlisle, Pa. 17013

FRIEDMAN, FRANK M., Dir. L. L., Flint Comm. Jr. Col., 1401 E. Court St., Flint, Mi. 48503

FURINO, PROF. VALERIA, 2103 West 12th St., Austin, Tx. 78703

FUSCO, JOSEPH F., Lab. Dir., Dept. Eng. For. Stu., St. Michael S. Col., Winooski, Vt. 15404

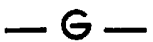

GABEL, DOROTHY, Ch. F D, Resurrection High School, 7500 W. Talcott, Chicago, Il. 60631

GALVIN, THOMAS F., Dir. For. Lang., Marshfield Pub. Sch., Marshfield, Ma. 02050

GIFFORD, NATHANIEL H., Head, Mod. Lang. Dept., Dir. Lang. Lab., Brooks School, North Andover, Ma. 01845

GAGNON, PROF. GILLES, Royal Military Col. of Can., Massey Bldg., Kingston Ont., CANADA 


\section{Membership Directory}

GALLASCH, H. F. JR., Dir. A-V Ctr., Davidson Com. Col., Bx. 1083, Lexington, N. C. 27292

GARDNER, LOIS, 3503 Robinhood Lane, Texarkana, Tx. 75301

GARFINKEL, ALAN, Tch., Dept. Educ. Assoc., Oklahoma State Univ., Stillwater, Ok. 74074

GARNER, MR. GARY N., ch FL, Dallas Baptist Col., P. O. Box 21206, Dallas, Tx. 75211

GARRISON, H. LESLIE, Hd. Dept. of Romance Lang., Trinity Junior Col., Langley B. C., CANADA

GARRUTO, MRS. FULVIAD, Red Bank H. S., Harding Rd., Red Bank, N. J. 07701

GASCHLIER, MICHAEL, 363 E. Gregson Ave., Salt Lake City, Ut. 84115

GATCHELL, ALICE, A P M L D, Webster Col., 470 E. Lockwood, Webster Groves, Mo. 63119

GATES, WARREN E., Chmn., Dept. For. Lang., Ohio Northern Univ., Ada, Oh. 45810

GAUDIN, LOIS S., Prof., Brooklyn Col. of Cuny, Dept. Mod. Lang., Brooklyn, N. Y. 11210

GAUDINO, V. A., LL Supv., Lakehead Univ., Oliver Rd., Port Arthur, Ontario, CANADA

GAUTHIER, J. H. LEON, R. I. Jr. Col., Mod. Lang., 199 Promenade St., Providence, R. I. 02908

GAUTIER, ROGER T., Lang. Lab. Dir., Univ. of Saskatchewan, Saskatoon Sask., CANADA

GEBAUER, PAUL, Asst. Prof., Linfield Col., 1324 Gilorr, McMinnville, Or. 97129

GEFFERT, CARL W., Assoc. Prof. of Ger., St. Andrew S. Presbyn. Col., Laurinburg, N. C. 28352

GEIGER, RICHARD A., Asst. Prof., Franklin and Marshall Col., Lancaster, Pa. 17604

GELINEAU, MRS. ANNE, Dir. Lang. Lab., Harvard U. G-4 Boylston HL, Cambridge, $\mathrm{Ma}, 02138$

GEMMATO, GENO R., Dir. Audio-Labs, Tufts Univ., Medford, Ma. 02155

GENDRON, RAYMOND, Head, Lang. Dept., Seekonk Sr. High School, Seekonk, Ma. 02771

GENNAULA, MRS. M. G., FL Chmn., Keystone Oaks HS, 1000 Kelton Ave., Pittsburgh, Pa. 15216

GEORGE J. PENNEY HIGH SCHOOL, 869 Forbes St., E. Hartford, Ct. 06108

GEORGIA, UNIV. OF, Dept. of German and Slavik Lang., Athens, Ga. 30601

GER. CTR., BOSTON, Dir. of Lang., Div., 170 Beacon St., Boston, Ma. 20116

GIANNETTI, GEORGE, Chmn. FL, Oak Park High School, 13701 Oak Park Blvd., Oak Park, Mi. 48237

GILBERT, SUSI, Dir. LL, Wofford Col., N. Church St., Spartanburg, S. C. 29301

GILL, MRS. SARA D., Mod. Lang. Dept., Scotland High School, Laurinburg, N. C. 28352

GINN AND COMPANY, Statler Bldg., 125 2nd Ave., Boston, Ma. 02154

GIONET, ARTHUR J., Lab. Dir., N. Texas State Univ., Denton, Tx. 76203

GIONET, BROTHER DENIS, Dir. LL, Scolasticat Central, 7000 Marie-Victorin, Montreal, CANADA 462

GLENNIE, VIOLA, Instr., Alpena Comm. Col., 666 Johnson St., Alpena, Mi. 49707 GOETZ, DONALD R., L L Dir., Lincoln High School, 1433 South 8th St., Manitowoc, Wi. 54220

GOLDELL, MARCUS, Prof. of Span., 251 Belmont St., Worcester, Ma. 01605

GOLDSTEIN, E. M., Assoc. Prof., Univ. of Ottawa, Dept. Ling. and Mod. Lang., 165 Waller St., Ottawa 2, Ontario, CANADA

GOLDSWORTHY, T. R., Dir. L. L., Univ. of Wis., 866 Van Hise Hall, 1220 Linden Dr., Madison, Wi. 53706

GOMEZ, FERNANDO, Ridgewood Ave., Ridgewood, N. J. 07451. 


\section{Membership Directory}

GONZALES, ANGELITA FLORO, California State Col., 27669 Loyola Ave., Hayward, Ca. 94545

GONZALEZ, MRS., Parra, Sp. Ir.s., Loretto Heights Col., 3001 S. Federal Blvd., Denver, Co. 80236

GOODRICH, ALBERT, A., Lang. Dir., Joint School Dist. No. 1, Three Lakes, Wis. 54562

GOODYEAR, STEPHEN, F L D H, Hull High School, 18C Main St., Hull, Ma. 02045

GORDON, ALAN M., Dir. of Lang. Lab., Univ. of Toronto, Toronto, 5 Ontario, CANADA

GORDON, DAN B., Grad. Dir., Dept. of Mod. Lang., Florida State Univ., Tallahassee, Fla. 32306

GORDON, DARRYL, Chmn. F. L., Springfield Pub. Schols, 511 Mill St., Springfield, Or. 97477

GORE, PETER B.. Lang. Lab. Dir., Deerfield Aca., Deerfield, Ma. 01342

GOULOT, SR. NOELLA. Notre Dame Col., 2321 Elm St., Manchester, N. H. 03104

GOWDA, MR. RAMAKRISHNA, "Ledar" S. C. A., 25 De Mayo 3433, Mar Del Plata, ARGENTINA

GOYER, DOREEN S., AP and LLS, Southwest Texas State Col., Mod. Lang. Dept., San Marcos, Tx. 78666

GRAMMATICOFF, ALEX, Ch. FL D, Florissant Valley Comm., 3400 Pershall Rd., Col., St. Louis, Mo. 63135

GRAY, MARY C., Supv. L L, Lenoir Rhyne Col., P. O. Box 279, Hickory, N. C. 28601

GREEN, JACK BYRON, Dir. For. Lang. Lab., Modesto Junior Col., Modesto, Ca. 95350

GREEN, ROBERT B. JR., L L Dir., West Va. Wesleyan, 48 Smithfield St., Buckhannon. W. Va. 26201

GREENE, ESTELLE S., Dir. LLR, Fairleigh Dickinson Univ., 207 Montross Ave., Rutherford, N. J. 07070

GREEN VALE SCHOOL, Glen Head Long Island, New York, N. Y. 11545

GREENWOOD, MRS. AUDREY, Chmn., Dept. of For. Lang., Idaho State Unive., Pocatello, Id. 83201

GREGORY, CLARA, Conslt., Texas Educ. Agency, 1202 Newning, Austin, Tx. 78704

GREIPP, JOSEPH F., 119 Sherwood Dr., Greenwood Pk., Blackwood, N. J. 08012

GRIFFIN, REV. J. J., Dir. Audio-Visual Lab., Allentown Col., Center Alley, Pa. 18034

GRIFFITHS, DR. D. A., Dep. Fr., Univ. of Victoria, P. O. Box 1700, Victoria, B. C. CANADA

GRIGG, BILLIE G.. L. L Sup., El Camino Col., 17204 Hass, Torrange. Ca. 90506

GRILLO. PAUL, Hd. Fr. Dept., North Country School. Lake Placid. N. Y. 12946

GRITTNER, FRANK, St. Supv./MFL, Wis. Dept. Pub. Inst., 710 Woodward Dr., Madison, Wi. 53704

GRONE, ELIZABETH, F. L. Cons., Lincoln Public Schools, Box 200, Lincoln, Nb. 68501

GROSJEAN, GLEN, Dir. LL, Univ. of Ca., B-40 Dwinelle Hall, Berkeley, Ca. 94720

GROSS, STEFANIA E., Lang. Lab. Dir., Bowling Green State Univ., Univ. Hall, Bowling Green, Oh. 43402

GROVER, M. ANSELM SR., Dir. LL Col. of the Holy Names, 3500 Mountain Blvd. Oakland, Ca. 94619

GRUNDSTROM, ALLAN W., Asst. Prof. of Fr., Bucknell Univ., Lewisburg, Pa. 17837

GUENETTE, PAUL G. Dir. Lang. Lab., Town of Brookline, Town Hall, 333 Wash. St., Brookline, Ma. 02146

GUERTIN, SISTER M. GEORGE, Mt. St. Mary Col., Hooksett, N. Y. 0310€

GUEST, DR. JAMES, Head. Lang. Dept., Pensacola Jr. Col., 1000 College Blvd., Pensacola, Fl. 32504 


\section{Membership Directory}

GUGGER, EDWARD M., Hd., Dept. of FL, Medford High School, Medford, Ma. 02155

GUNDLACH, C. M., Supv. of LL, Wilmington Col., Box 990 Col. P O, Wilmington, Oh. 45177

GURA, ROSALYN, L L Supv., Youngstown State Univ., 410 Wick Ave., Youngstown Oh. 44503

GUTTIERZ, RONALD R., J. I. Case High School, Racine. Wi. 53406

GUZEK, KATHLEEN J., Fr. Tchr., Ontonagon Area High School, 304 Pennsylvania Ave., Ontonagon, Mi. 49953

GUZMAN, ERNESTO A., Coor. Mod. FL, Tarrant Co. Jr. Col., South Campus, Ft. Worth, Tx. 76119

\section{$-\mathrm{H}-$}

HAGEN, MRS. DONALD F., Dir. Lang. Lab., Fairleigh Dickinson Univ., 285 Madison Ave., Madison, N. J. 07940

HAGUE, MORTON, P. O. Box 10104, Oakland, Ca. 94610

HALENZ, ESTER, Chmn., Columbia Union Col., Takom Park, Me. 20012

HALL, LEWIS, Aca. Dean, Lees-McRae Col., Banner Elk, N. C. 28604

THE HALTON COUNTY BD. EDUC., P. O. Box 548, Oakville, Ontario, CANADA

HAMMOND, JOHN H., Ch., Texas Christian Univ., Dept. of For. Lang., Ft. Worth, Tx. 76129

HAMSON, ERWIN M., Dir., Lang. Lab., Univ. of Michigan, 1401 Mason Hall, Ar:n Arbor, Mi. 48104

HAND, RAYMOND, Inst. F L, Alexander City State, Junior Col., Alexander City, Al. 35010

HANSON, DAVID A., A P R, Ger. and Slavic Lang., Brandeis Univ., Waltham, Ma. 02154

HANSON, JAMES, Chmn. Dept. of Mod. Lang., Adrian Col., Mahan Hall, Adrian, Mi. 49221

HARDING, JACOBINA, Head FL Dept. and LL.supvsr., Alamo Hts. H. S., 6900 Broadway, San Antonio, Tx. 78209

HARE, THOMAS J. O., U. of Texas, Lang. Lab., Batts Hall 227, Austin. Tx. 78712

HARRISON, BR. EDW.ARD, Fr. Sp. Inst., Walsh Col., 2020 Easton Rd., Canton, Oh. 44720

HARTLEY, G. CHRISTOPHER, Head Lang. Dept., The Peddie School, Hightstown, N. J. 08520

HARTMAN, ALICE, Chmn. FL, Dept., Joliet Twp. H. S. West, 401 N. Larking, Joliet. Il. 60435

HASENCLEVER, MISS IRENE, Lang. Lab. Dir., Bennington Col., Bennington, Ct. 05201

HAUGHTON, V., Chmn. Fr. Dept., McDuffie School, Sprir.gfield. Ma. 01107

HAWKINS, PAUL JR., A Ger. Chmn., W. Va. Univ.-Parkersburg Ctr., 110 16th St., Vienna, W. Va. 26101

HAYASE, MISS YURIKO, Dir. Lang. Lab., Winthrop Col., Rock Hill. S. C. 29730

HAYES, PAUL H., D H F L, Framingham No. High Schnol, A-Street, Framingham, Ma. 01701

HAYWARD, EARL, F', Dir. Lang. Lab., Alma Col., Alma, Mi. 48801

HEADQUARTERS, DLIWC Aca. Libr.. Bldg. No. 302, Presidio Mntry, Ca. 93940

HEDERVARY, CHARLOTTE, Konth Tea., 3412 Main St., College Park. Ga. 30337

HEERMANS. LILLIAN, Dir. Lang. Lab., Vassar Col., Poughkeepsie, N. Y. 12600

HEFKE, PROF. GEORGE W., Asst. Prof. of Lang., Kansas Wesleyan, Salina, Ka. 64701

HEGGOY, KORE N., Dir. Lang. Lab., Randolph-Maccn Col., P. O. Box 726, Ashland, $\mathrm{Va} .23005$

HEIMAN, MRS. MONICA, West Texas State Univ., Canyon, Tx. 79015

HEINE, HEINRICH, Supv. Lang. Lab., American Univ., 3004 Woodlawn Ave., Falls Church, Va. 22042 


\section{Membership Directory}

HEPBURN, ERIC, Chmn. For. Lang., 335 Wadsworth Ave., New York, N. Y. 10040 HERNANDEZ, ADELE B., Instr. of Span., 96 Falmouth St., Portland, Me. 04103 HERON, ROBERT F., FL Dep. Hd., Bellingham H. S., Bellingham, Ma. 02019

HERRERA, LAZARO M., LL Dir., The Westminster Schools, 1424 W. Pace Ferry Rd. NW, Atlanta, Ga. 30327

HERTJE, ARVELLA, LL Dir., Instr. Span., St. Ambrose Col., Davenport, Ia. 52803

HESKAMP, DR. H. READE, Dir. FL Lang., MacMurray Col., 143 Caldwell, Jacksonville, Il. 62650

HESSLER, SR. GRETCHEN, OP Lang. Lab. Dir., Rosary Col., River Forest, Il. 60305

HEUBLEIN, LINDA, Dir. L L, Col. of St. Teresa, Winona, Mn. 55987

HEUERMAN, SHARON, Lab. Supv., Woodstock High School, Woodstock, Il. 60098 HEWSON, DR. J., Mem. Univ. of Newfoundland, St. John S NFL CANADA

HICKMAN, CHARLES H. JR., 4025 8th St. SO, St. Petersburg, Fl. 33705

HICKS, DAVID B., Head ML Dept., The Pingry School, North Ave., Hillside, N. J. 07207

HICKS, ROBERT Z., Instr. in Speech, Univ. of Hawaii, Hilo Campus, Hilo, Ha. 96720

HILL, REV. FATHER SEBASTIAN, Chmn.-Mod. Lang., Father Judge Seminary, Monroe, Va. 24574.

HILTS, MRS. MARGARETE, Loma Linda Univ., Riverside, Ca. 92505

HINES, ELIZABETH, 16588 Oleander Ave., Los Gatos, Ca. 95030

HINKLE, RUTH C., Dir. L L, Davis and Elkins Col., 106 Buffalo St., Elkins, W. Va. 26241

HINTLIAN, MRS. S., FL D Ch., Enfield High School, Enfield St., Thompsonville, Ct. 08082

HIRSCH, MRS. BERNICE, D L L, Samford Univ., 800 Lakeshore Dr., Birmingham, Al. 35209

HIRTH, DAVID R., P. O. Box 35, Deerfield, Ma. 01342

HOCKING, ELTON, Mod. Lang. Educa., Purdue Univ., Coulter Hall, Lafayette, In. 47907

HOFFER, MRS. PAMELA, 4 Ridge Rd., Lincoln, Ma. 01773

HOLLAND, REUBEN W., Dir. Lang. Lab., Univ. of Tennessee, Chattanooga, Tn. 37403

HOLLINS COL., Lang. Lab. Dir., Hollins Col., Va. 24020

HOOT, JAY, Ger. Instr., Northland Col., Ashland, Wi. 54806

HOPKENS, A. H. JR., Fr. and Eng., Middlefield Mem. Jr. High, Middlefield, Ct. 06455

HOPKINS, AIRED H. JR., Fr. Tea., 80 Strong Rd., Middlefield Mem. Jr. High, S. Windsor, Ct. 06074

HOPPEL, MISS ALICE, 32nd and Anza Sts., San Francisco, Ca. 94121

HOULIHAN, ROBERT, Dir. Eng. Lang. Inst., Tokyo, 15-9-3-Chome Higashi Ikeb, Tokyo, Japan

DIRECTOR, For. Lang. Dept., Sam Houston State Col., Huntsville, Tx. 77340

HOWELL, NORA C., Lang. Lab. Dir., Appalachian State Univ., Boone, N. C. 28607

HOWELL, RAYMOND, Dir. A-V, 2202 Rucker Ave., Everett, Wa. 98201

HOWLING, ROBERT T., Chmn. Eng. Hum., New Haven Col., New Haven, Ct. 06505

HUBBARD, DR. LOUISE J., Chmn. D. C. Tea. Col., Div. of For. Lang., 11th and Harvard Sts. NW, Washington, D. C. 20009

HUFF, MRS. JEAN, 7525 Newton, Overland Park, Ka. 66204

HUFFMAN, DONALD T., Prof., W. Va. Univ., 781 Meadowbrook Rd., Morgantown, W. Va. 26505

HUGHES, EUGENE E., Chmn. Div. of Lang. Arts, Southwestern State Col., Weatherford, Ok. 73096

HUGHES, WILLIAM H., Instr., Col. of Southern Idaho, P. O. Box 1238, Twin Falls, Id. 83301 


\section{Membership Directory}

HUGOT, FRANCOIS, Lect., Brown Univ., Box E, Providence, R. I. 02912

HULL, ALEX P. JR., Tex Tech. Col., Dir. L. L., F. O. Box 4285, Lubbock, Tx. 79409 HULST, VAN SISTER KATHRYN, Span. Tea., Columbus High School, Marshfield, Wi. 54449

HUMPHREY, HARVEY J. JR., Assoc. Lang. Lab. Dir., Fordham Univ., Bronx, N. Y. 10458

HUNDLEY, MRS. MARY ELLEN, Dept. Chmn. For. Lang., 405 36th Ave. N., Seattle, Wa. 98102

HUTCHINSON, DR. JOSEPH C., HG Defense Lang. Inst., 502 G. St. SW, Washington, D. C. 20024

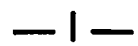

IANNUZZI, PROF. D. A., Ch. Mod. Lang. Dept., Niagara Univ., F. O. Box 164, Niagara Univ., N. Y. 14109

IBBOTSON, A., Dir. Mod. Lang. Audio-Visual Ctr., Queen's Univ., Kingston, Ontario, CANADA

INES, SR. MARIA, Dir. L. L., St. Joseph S. Col. for Wmn., 245 Clinton Ave., Brooklyn, N. Y. 11205

INGERSOLL, J., Tea., Sr. High School, Grand Rapids, Mn. 55744

INSTRUCTIONAL RESOURCES CENTRE, State Univ., of N. Y., Stony Brook, N. Y. 11790

IOUP, L. GEORGETTE, Stu., NYU, 402 W. 22nd St. N 3F, New York, N. Y. 10011

IRVING, DR. EVELYN U., Carson-Newman Col., Jefferson City, Mo. 65101

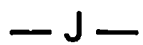

JABLON, DR. KENNETH, Prof. and Hd. of MFL, Frostburg State Col., Frostburg, Md. 21532

JACKSON, GORDON, Lang. Lab. Dir., Capital Univ., 1819 Rosehill Rd., No. 15, Reynoldsburg, Oh. 43068

JACKSON, JAMES W., L. L. Dir., Western Mich. Univ., Dept. Mod. Lang., Kalamazoo, Mic. 49001

JACKSON, MARY E., Dir. For. Lang. Lab., Morgan State Col., Baltimore, Md. 21212

JACQUARD, SANFORD E., 241 Moreno Dr., Beverly Hills, Ca. 90212

JACQUES, FERNAND, Instr., Lakewood High School, 13429 Emerson Ave., Lakewood, Oh. 44107

JACKSON, DR. RICHARD W., Coord. FL-Palm Beech County, 3323 Beleverde Rd., W. Palm Beech, Fl. 33401

JALLING, HANS, Dir., LL Research Project, U. Stock-Strandbacken, Bromma, Sweden

JANES, JOSEPH J., Hinsdale High School, 7300 Clarendon Hills, Clarendon Hills, Il. 60514

JARLETT, FRANCIS G., Dir. Lang. Lab., Central Ct. State Col., New Britain, Ct. 06050

JEAN, SR. MARY, OP, CH FLD, Barry Col. Miami. Fla. 33161

JERNER, CARL, 2600 M. Bell Dr., San Pablo, Ca. 94806

JERNER, CARL, 2600 Mission Bell Dr., San Pablo, Ca. 94806

JESPERSEN, JOHANNES, R. I, Jr. Col. Mod. Lang., 199 Promenade St., Providence, R. I. 02908

JOBIN, JACQUES, Tea., St. Dominic Col., 1405 N. Fifth Ave., St. Charles, Il. 60174

JOCHMANS, ROBERT P., Chmn. Mod. Lang. Dept., Union Col., Lincoln Nb. 68506

JOHNGREN, RUTH M., Head FL Dept., Norwood Senior High School, Norwood, Ma. 02062

JOHNSON, BARBARA, Wa. State Univ., For. Lang. Dept., Pullman, Wa. 99163

JOHNSON, ROBERTA, Dir. Lang Lab., Claremont Men S. Col., Claremont, Ca. 91711 


\section{Membership Directory}

JOHNSON, MRS. VERONA, Lang. Lab. Aide, 4843 North 51 St., Milwaukee, Wi. 53218

JONES, A. JAMES, Pa. State Univ., S-320 Burrows Bidg., University Pk., Pa. 16802 JONES, MRS. AMY K., 731 E. Main St., Jackson, Oh. 45640

JONES, R. J., The Col. of Ed., Univ. of Toronto 70, 371 Bloor St., W., Toronto 5, Ontario CANADA

JONG, THEODORE DE, FL Inst., N. Orange City Jr. Col. Dist., P. O. Box 3633. Fullerton, Ca. 92634

JOSEPH, ST. ACADEMY, 3200 Grand Ave., Des Moines, Ia. 50312

JUAN, M. SR., Ch. Fr. Dept., Regis Col., 235 Wellesley St., Weston, Ma. 02193

JUHEL, DENIS N. F., Dept. Romance Lang. UNB, Fredericton NB, CANADA

JULIE, SR., Hd. Fr. Dept., Maryvale-Trinity Col. Prep School, Valley Rd., Brooklandville, Md. 21022

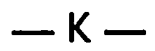

KADAR, DR. G., Dir. of Lang. Lab., Napa Col., Dept. of FL, Napa, Ca. 94558

KAPLAN, D., For. Lang. Coord., Cold Sprg. Harbor High Sch., Cold Sprg. Harbor, N. Y. 11724

KAUB, SHIRLEY J., P. O. Box 2189, 545 W. Dayton St., Madison, Wis. 53703

KAWARABAYASHI, YUSUKE, Dir., Univ. of Pacific, 145 Bernice Ave., Stockton, Ca. 95207

KAY, M. L., York Univ., Dir. Div. Ling. and Lang. Trng., Dowrsview, Ontario, CANADA

KEENAN, MISS P., 2682 17th Ave., San Francisco, Ca. 94116

KEENEY, JANET, For. Lang. Tea., P. O. Box 744, Madras, Or. 97741

KEHOE, DENNIS, Lang. Lab Dir., Wi. State Univ., Whitewater, Wi. 53190

KEHOE, RITA, Asst. Lang. Lab. Dir., Wi. State Univ., Route 2, Whitewater, Wi. 53190

KEK, DR. ANNA DALE, Ch. Lang. Dept., David and Elkins Col., Elkins, W. V. 26241

KELLER, J. P.. Chmn., Dept. Mod. Lang., Albion Col., Albion, Mi. 49224

KELLINGER, MRS. CESI, Lab. Dir., Wilson Col., Chambersburg, Pa. 17201

KELSO, SISTER BETH, Lang. Lab. Dir., St. Mary of Woods Col., St. Mary of Woods, In. 47876

KELSO, PRISCILLA, Dir. Lang. Lab., Newton Jr. Col., Washington Park, Newtonville, Ma. 02160

KEMP, MR. LINWOOD S., Lang. Lab. Tech., Westfield State Col., Westficld, Ma. 01085

KEMPRECOS, JOSEPH, LLD, Rumson Fair Haven Reg Hi, Rumson, N. J. 07760

KENNEALY, WILLIAM P., Lexington High School, 7 Hancck Ave., Lexington, Ma. 02173

KENNEDY, DAPHENE, Supv. L. L., Dept. of Mod. Lang., Freed-Hardeman Col., Henderson. Tn. 38240

KENT, SR. MARY KATHERINE, Ch., Mod. For. Larig. Dept., 103rd. and Ceritral Park Ave., Chicago, Il. 60655

KERR, CHARLOTTE W., Supv. L L, Occidental Col., $1 € 00$ Campus Rd., Los Angeles, Ca. 90041

KHOURY, DWIGHT, Dept. of Mod. Lang., Keuka Col., Keuka Park, N. Y. 14478

KIBART, MRS. SYLVIA, FL Dept. Etsu, Commerce, Tx. 75428

IIEKEL, R. D., Asst. Prof., Dept. of Mod. Lang., Or. State Univ., Corvallis, Or. 97331

KIMMEL, DR. ARTHUR S., For. Lang. Dept., Western Washirgton State Col., Bellingham, Wa. 98225

KIRBY, MRS. CAROL, 922 Swift Ave., Sheboygan, Wi. 53081

KIRKCONNELL, PROF. THOMAS, Chmn., Dept. of Mod. Lang., W. Va. Inst. of Tech., 114 4th Ave., Montgomery, W. V. 25136 


\section{Membership Directory}

KIZER, WALLACE L., DLL, Baptist Col. at Charleston, P. O. Box 10087, Charleston, S. C. 29411

KLEINSASSER. J. A., Hd. Lang. Dept., Sioux Falls Col., Sioux Falls, S. D. 57101

KLOSOWSKI, THOMAS H., Tea., Montville H. S., Oakdale, Ct. 06370

KNUDSEN, JOHN K., Dir., Lang. Lab., Univ. of Pittsburgh, Dept. of Gen. Ling., Pittsburgh, Pa. 15213

IKOEHLER. GEORGE E., Lang. Lab. Dir., St. Procopius Col., Lisle, Il. 60532

KOENEN, SHIRLEY, Instr. of Fr., 630 18th Ave., Lewiston, Id. 83501

KOPP, DR. W. LAMARR. Asst. Dean, Pa. State Univ., 138 Sparks Bldg., Uni. versity $\mathrm{Park}, \mathrm{Pa} .16802$

KOPPENHAVER, JOHN, Hd. of Sp. Dept., Hesston Col., 445 S. Main St., Hesston, Ks. 67062

KORIYAMA, S., Sony Corp. of America, 47-47 Van Dam St., Long Island City, N. Y. 11101

KOVARY, PROF. TOM T., LL Dir., State Univ. of N. Y., Col. at Cortland, Cortland, N. Y. 13045

KRAIL, JACK B., Audio Labs., Norfolk State Col., 2401 Corprew Avc., Norfolk, Va. 23502

KRAMM, DALE F., Instr. FL, Col. of St. Thomas, 1295 Blair Ave., St. Paul, Mn. 55104

KRUSE, MR. EDWARD L. JR-., Lect. in Educ., Canisius Col., Buffalo, N. Y. 14208

KRYNITZ, ROY L., Lab. Dir., For. Lang. Dept., New Mexico State Univ., University Park, N. M. 88001

KUCHAU, PAUL F., 215 McKay Bldg., Brigham Young Univ., Provo, Ut. 84c01

KUEI, SYLVIE, Canby St. 416, (Co Christensen), Vermillion, S. D. 57069

KULTURA, Newspr. Import Co., Egyetemi Konyvtar, Pob 483, Budapest. Hungary

KUMP, RICHARD, Southeast Mo. State Col., 337 Themis, Cp Girardeau, Mo. 63701

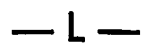

LABELLE, QUENTIN, Windsor Mountain School, Lenox, Ma. 01240

LABROSSE, ANDRE, Lang. Dir., Windsor Locks Jr. High, Windsor Locks, Ct. 06096

LACEFIELD, ARCH. S., Sr. Instr., Col. Lib. Univ. of Ky., Henderson Comm. Col.. Henderson, Ky. 42420

LACKAU, PAUL, Brigham Young Univ., Lang. Lab. 215 McKay Blc'g., Provo, Ut. 84601

LAFAURY, PH G., LL Dir., Univ. of St. Michael S. Col., 50 Sr. Joseph Sr. Fr. Dept., Toronto 5 Ontario. CANADA

LAFFERTY, BRO. RICHARD, LaSalle H. S., 8605 Cheltenham Ave., Philadelphia. Pa. 19118

LAFFORD, L. A., Dir. of Audio-Visuals, St. Mary S. Univ., Robie St., Halifax, N. S. CANADA

LAFOREST, SR. ELIZABETH, Pr. Fr., Mercy Col. of Detroit, 8200 W. Outer Dr., Detroit. Mi. 48219

LAGUEX, RAYMOND J., Act. Chmn. Dept. of Lang., Farmirgton State Col., Farmington, Mc. 04938

LALLY, DALE V., Dir. L. L., Marquette Univ., Milwaukee, Wi. 53233

LANDER, MARK C., Saltaire Dr.., Old Lyme, Ct. 06371

LANDRY, SISTER MONICA, Notre Dame HS, 910 N. Easton Ave., Crowley, La. 70526

LANGE, DALE L., Assoc. Prof., Univ. of Minnesota, 152 ^ Peik Hall, Minneapolis. Mn. 55455

LANGE. JOHN B., Assoc. Prof., St. Benedict S. Col., 526 Div., Atchison Ks. 66002

LANGLEY, TASSIE REE, A P E, Atlantic Christian Col., Dept. of Eng., Wilson N. C. 27893 


\section{Membership Directory}

LOUBERT, SISTER ELAINE, Col. of St. Scholastica, Duluth, Mn. 55811

LOWENTHAL, ROBERT, Dir. Lang. Lab., Wesleyan Col., Rocky Mount, N. C. 27801

LUBIC, MICHAEL K., Asst. Prof., Miles Col., Birmingham. Al. 35208

LUBS, MARGARET, Armstrong State Col., Savannah, Ga. 31406

LUCAS, Boyce M., Dir. Lang. Lab., Whitehall High School, Whitehall, Mi. 49461

LUCAS. LEONARD, 2355 Hunter Mill Rd., Vienna, Va. 22180

LUCKAU, PAUL F., Asst. Prof., Brigham Young Univ., 215 McKay Bldg., Provo, Ut. 84601

LUIKART, TIM, Lang. Lab. Dir., Ashland Col., Ashland, Oh. 44805

LUJAN, FRANK JR., Tea., W. Las Vegas High, 1327 Fifth St., Las Vegas, N. M. 87701

LUNDERVILLE, GERALD P., L I, Cape Cod Comm. Col., 367 Main St., Hyannis, Ma. 02601

LYNCH, MRS. ANNA, Dir. Lang. Lab., Trinity Col., Washington, D. C. 20910

LYTLE, MRS. B. G., Asst. Lab. Tech., Onondaga Comm. Col., Rd. No. 2, 67 Warren St., Tully, N. Y. 13159

LYTTON, MRS. VEGA, Lang. Lab. Dir., Mary Baldwin Col., Staunton, Va. 24401

MACADOO, T. O., AP and Dir. Lang. Lab., Dept. of For. Lang., VA Polytechnic Inst., Blacksburg, Va. 24061

MACHEY, LOLA A., 8731 E. Bonnie Rose, Scottsdale, Az. 85251

MACINKO, JOHN, Dir. FL Lab., Old Main Bldg., U. of Colorado, Boulder, Co. 80300

MACKIN, PATRICK, Hd. Master, Keith Country Day School, 1715 N. 2nd St., Rockford, Il. 61107

MADDUX, W. K., Assoc. Prof. Fr., Carthage Col., Kenosha, Wis. 53140

MADELEINE, SR. MARY, P. of Fr., Col. of Notre Dame of MD, 4701 N. Charles St., Baltimore, Md. 21210

MAESTAS, MARTIN M., 5570 Winchelsea Dr., Normandy, Mo. 63121

MAGDALEN, SR. MARIE, OP, Lang. Lab. Dir., Rosary Col., River Forest, Il. 60305

MAGILL, DR. ROBERT, Dir., Lang. Lab., Towson State Col., Dept. Mod. Langs., Baltimore, Md. 21204

MAHLER, MICHAEL, Dir. LL, Univ. of Mo., 8001 Natural Bridge, St. Louis, Mo. 63121

MALI, PIERCE, ML Hd., Princeton Day School, The Great Rd., Princeton, N. J. 08540

MANDEL, E. JULES, Coord., Supv. Tchg. at UCLA, 20918 Calimali Rd., Woodland Hills, Ca. 91364

MANN, G. THOMAS, Instr. of Ger., Graceland Col., Lamoni, Ia. 50140

MANNING, SR. MARGARET, Dir. Lang. Lab., Notre Dame Col. of S I, 300 Howard Ave., Staten Island, N. Y. 10301

MANOLIS, JOHN C., L. L. Dir., Mary Wash. Col. of U. if Va., P. O. Box 1126, Col. Sta., Fredericksburg, Va. 22401

MANWELL, RACHEL, L L Asst., Univ. of Il., 215 Lincoln Mall, Uubana, Il. 61801

MAPA, SR. M. V., LL Dir., San Francisco Co. for WMN, Lone Mt., San Francisco, Ca. 94118

MARCELL, NOAH, FL Chm., C. L. Community HS, 45 West Franklin St., Crystal Lake, Il. 60014

MARKEL, DOROTHY, Asst. Prof. of FL, Newark St. Col., Morris Ave., Union, N. J. 07083

MARSHALL, PROF. D. W., Vancouver City Col., 912 West 12th Ave., Vancouver 9, BC, CANADA

MARSHALL, ROBERT T., Chmn., Dept. of Lang. and Ling., Mt. St. Mary S R D 1, Emmitsburg, Md. 21727 


\section{Membership Directory}

LANIAK, MISS M., Asst. Dir., Rm. 2 Asbury N / LL OPS, Amer. Univ.-Nebr and Mass, Washington, D. C. 20016

LANNUZZI, PROF. D. A., Chmn. Mod. Lang. Dept., Niagara Univ., P. O. Box 164, Niagara Univ., N. Y. 14109

LAPP, DONALD, Prof. Sp., Aurora Col., 347 Gladstone, Aurora, Il. 60507

LARKIN, JAMES B., Asoc. Prof. Span., Coe Col., Cedar Rapids, Ia. 52402

LARSON, ELINOR C., Lab. Dir., Scotch Plains-Fanwood H. S., Westfield Rd., Scotch Plains, N. J. 07076

LARSON, L. C., Asst. Dean, Audio Visual Dir., In. Univ., Bloomington, In. 47401

LASATER, HELGA D., Teacher's Aide, Montrey Penisula Col., 491 Hawthorne St. No. 8, Monterey, Ca. 93940

LASHUA, MARGARET, Dir., For. Lang., Glastonbury Pub. Schools, Gideon Welles Jr. High School, Glastonbury, Ct. 06033

LATORRACA, JOSEPH P., 64-B Taylor Ave., E. Brunswick, N. J. 08816

LAURENTIAN UNIV., Lang. Lab., Sudbury, Ontario, CANADA

LAWLOR, JOSEPH P., LL Dir., Lake Forest High School, 1285 N. McKinley Rd., Lake Forest, Il. 60045

LAWSON, DONALD W. G., Dir., Paine Col., A-V Dept., Augusta, Ga. 30901

LAY, AMADO M., Prof. Span., Gustavus Adolphus Col., Zox 1413, St. Peter, Mn. 56082

LEAHY, S. PETER, Lab Dir., Wis. State Univ., Stevens Point, Wi. 54481

LEBOEUF, JANINE, Charlton High School, Upper Gore Rd., Webster, Ma. 01570

LEE, MARIE LORRAINE, Fr. Dept., Far Hills City Day School, Far Hills, N. J. 07931

LEIGHTON, CHARLES, Ch. Dept. Span. and Classics, A P, Univ. of New H., Durham, N. H. 03824

LEONE, ARTHUR A., 2903 Shelly Dr., Indiana, Pa. 15701

LEVINSON, MRS. B., Supv. Lab., Brooklyn Col., City U. Bedford Ave. and Ave. H, Brooklyn, N. Y. 11210

LEWIS, DAVID W. P., Lakehead Univ., Port Arthur, Ontario, CANADA

LEWIS, MRS. MARSHA, Tompkins-Cortland Comm. Col., 175 Main St., Groton, N. Y. 13073

LEWIS, MISS WYNELLE, Hd. Span. Dept., Dir. L L, Peace Col., Raleigh, N. C. 27602

LIEBIG, GLEN, L L Dir., Instr. Span., Bryan Col., Dayton, Tn. 37321

LIGHT, RICHARD L., Lab. Dir., Dept. Eng. For. Stu., St. Michael S. Col., Winooski, Vt. 15404

LIGHTCAP, DAVID W., Audio-Visual Dir., Susquehanna Univ., Selinsgroe, Pa. 17870

LINDBERG, R. H., Chmn. Lang. Dept., Gov. Dummer Aca., Bayfield, Ma. 01922

LINDNER, LUIS. Dir. Lang. Lab., Stephens Col., Columbia. Mo. 65203

LINDQUIST, MRS. PIRKKO, Alex von Humboldt Kielil, Turun Ylipoisto-Turku, Suomi, Finland

LINDNER, LUIS, Dir. Lang. Lab., Stephens Col., Columbia, Mo. 65203

LINGUAPHONE INSTITUTE, 207-209 Regent St., London, England

LIST, DR. EDGAR A., Chmn. Dept. of For. Langs., Fresno State Col., Fresno, Ca. 93726

LOMBARDO, S. JOSEPHINE, Rosary Hill Col., 4380 Main St., Buffalo, N. Y. 14226

LONG, VERNON M., Otero Junior Col., P. O. Box 779, La Junta, Co. 81050

LOOMIS, RUTH, FL Curr. Assoc., Madison Memorial H S, 201 S. Gammon Rd., Madison, Wi. 53705

LORD BEAVORBROOK H. S., Hd. of Fr. Dept., 9019 Fairmount Dr. SE., Calgary 30, Alberta, CANADA

LO RE, DR. A. G., Dir. Lang. Lab., Univ. of N. Carolina, 105 Dry Hall, Chapel Hill, N. C. 27514

LOTTMANN, ANNA MARIE, Prof., Meramec Comm. Col., 959 S. Geyer Rd., Kirkwood, Mo. 63122 


\section{Membership Directory}

MARSHALL, WILLIAM H., Asst. Prof. of Lang., Univ. of Ma., Augusta, Me. 04330 MARTIN, BRO. JAMES, CSC, Holy Cross High School, 26-20 Francis, Lewis BL, Flushing, N. Y. 11358

MARTIN, WILLARD M., DLL, Pa. State Univ., 6 Sparks Pa. State Univ., Univ. Park, Pa. 16802

MARTINEZ, CARLOS CORTEZ, P. O. Box 1147, Bilbzo-Vizcaya, SPAIN

MARTINEZ, JOHN, CH SP Dept., New Trier Twp. H. S. W., 7 Happ Rd., Northfield, Il. 60093

MARTINEZ, ROGELIA R., D. L. L., Morris Brown Col., 914 Gaylemont Circle, Decatur, Ga. 30033

MARTINEZ, DR. R. V., Chmn. Span. Dept., Franklin and Marshall Col., Lancaster, $\mathrm{Pa} .17601$

MARTINI, PROF. PADOUE DE, Dir. Lang. Lab., Univ. of Ca., Los Angeles, Ca. 90024

MARXHEIMER, EDWARD, Lang. Lab. Dir., Univ. of Alberta, Edmonton, Alberta, CANADA

MASON, GEORGE, Educational Electronics Inc., P. O. Box 422, Watertown, Ma. 02172

MATRANGA, EDWARD C., Supv. of For. Lang., Stratford Town Schools,, Stratford, Ct. 06497

MATURE, DR. A. P., Head-Lang. Dept., Newberry Col., Newberry, S. C. 29108

MATTHEWS, HELENE, Dir. Lang. Lab., Adekphi Suffolk Col., 89 Collins Ave., Sayville, N. Y. 11782

MAXWELL, WILlIAM C., Head Lang. Dept., Tabor Aca., Box 372, Marion, Ma. 02738

MAYS, MRS. RUTH ANN, Prof. of Fr., Pikeville Col., Pikeville, Ky. 41501

MAZZA, BRO. R., CFX, St. John S. HS, 378 Main St., Shrewsbury, Ma. 01545

McCLINTOCK, GEO. L. M. Jr., 1825 McLaughlin Run Rd., Pittsburgh, Pa. 15241

McCONOCHIE, DR. J. A., Lab. Dir., N. Y. Univ., 435 East Bldg. NYU, New York City, N. Y. 10003

McCULLOUGH, MR. J. T., Sacramento, St. Col., Sacramento, Ca. 95819

McDONALD, MRS. PEARL S., Supvr. For. Lang., 1426 N. Quincy St., Arlington, Va. 22207

McGETTRICK, HELEN, 115 St. Stephen St., Boston, Ma. 02115

McGIRT, PAUL B., Clark Col., 240 Chestnut St. SW, Atlanta, Ga. 30314

McKENNA, J., Lang. Lab. Dir., Col. of the Holy Cross, Worcester, Ma. 01510

McKENNA, MRS., Sec., Hyde School, Bath, Me. 04530

McKNIGHT, M. W., Assoc. Dir. Lang. Lab., Univ. of Ca., Irvine, Ca. 92664

McLANE, JEAN, Asst. Prof., McPherson Col., McPherson, Ks. 67460

McMULLAN, JOHN, Dir. Lang. Lab., St. George S. School, Newport, R. 1. 02840

McNAMARA, MRS. M. J., Supv., Lang. Lab. Arts Bldg., Douglass Col., New Brunswick, N. J. 08903

MCTIGUE, DR. T. M., Chmn. Dept. For. Lang., Bellarmine Col., Louisville, Ky. 40205

MEGOW, GERHARD., Prof., Hope Col., Holland, Mi. 49423

MEIER, MR. WALTER, Seebacherstrasse, 8052 Zurich, Switzerland

MERCEDES, SISTER IRMA, Instr., Chestnut Hill Col., Philadelphia, Pa. 19118

MERCHANT, ROBERT C., Quality Electronic System, P. O. Box 246, Carmel Valley, Ca. 93924

MERCHANT, ROY, PMB 3045, Kano, Nigeria, AIR MAIL

MERKENSTEIJM, E. C. VAN, AD/Lang. Lab., 320 Logan Hall, Univ. of Pa., Philadelphia, $\mathrm{Pa} .19104$

MERZ, ELIZABETH S., Ch. D I, Peabody Conserv. Music, 818 Park Ave., Baltimore, Md. 21201

MESS, MARY JANE, Ch. FL Dept., Ottowa Typ. H. S., 211 E. Main St., Ottawa, Il. 61350 


\section{Membership Directory}

MESSNER, C. A., Assoc. Prof. Mod. Lang., Carleton Col., Northfield, Mn. 55057 METFORD, DR. JACQUES, Dir. Univ. Lang. Labs., Univ. Col. UWO, London Ontario, CANADA

METSCH, PROF. O. F. JR., C F L, Worcester Junior Col., 766 Main St., Worcester, Ma. 01608

METT, F. B., Inst., Res. at Bay, 1720 E. Capitol View HS, Milwaukee, Wi. 53211

METTLER, DONALD G., Lang. Lab. Dir., Edison Twnsp. H. S., South Colton Rd., Edison, N. J. 08817

METZGER, ELOISE, Lang. Coord., Pekin Comm. High School, 1502 James Rd., Perkin, Il. 61554

MEYER, M. D., Lab. Dir., Hamline Univ., German Dept., St. Paul, Mn. 55101

MICKO, MICHAEL, Lang. Lab. Dir., PMC Col., Chester, Pa. 19013

MICOZZI, ARTHUR, Supv. F. L., Board of Edu., Balti Co., 2401 Lampost Lanr, Baltimore, Md. 21234

MIDDLETON, R. T., 3rd. Dir. Lang. Lab., Tougaloo Col., Tougaloo, Ms. 39174

MIELE, HARRY, Lang. Dept., Allegany Comm. Col., 340 Frederick St., Cumberland, Md. 21502

MIKESELL, NORMAN, Dir. L. L., Ind. Univ., Ballantine Hall, Rm 120, Bloomington, In. 47401

MILES, DR. E. KENNETH, Prof. in Ger., Univ. of Me., 214 Little Hall, Orono, Me. 04473

MILLER, DEAN, Univ. of Co., 1416 3rd. St., No. C, Boulder, Co. 80302

MILLER, DOROTHY A., 3151 S. Dahlia, Denver, Co. 80222

MILLER, EUGENE, Cncl. Mod. Lang., St. U. of NY at Buffalo, 250 Winspear Ave., Buffalo, N. Y. 14214

MILLER, J. B., Wittenberg Univ., Springfield, Oh. 45501

MILLER, J. DALE, A P F and I, 360 McKay Bldg., Brigham Young Univ., Provo, Ut. 84601

MILLER, MRS. H. W., Dir. Lang. Lab., Carson.Newman Col., Buckingham Dr., Rt., Jefferson City, Tn. 37760

MILLER, MARTHA R., Sec. to Dir., Harvard U. of Mod. Lang. Ctr., Boylston Hall G3 Harvard, Cambridge, Ma. 02138

MILLER, MYRON C., Prof. Ger., Polk Junior Col., 999 Ave. "H" NE, Winter Haven, Fl. 33880

MILLER, ROBERT C., Lang. Dept. Chrm., Thatcher School, Ojai, Ca. 93023

MILLER, DR. ROYCE W., Chmn., M. L. D., Gordon, Col., 255 Grapevine Rd., Wer:ham, Ma 01984

MILLER, SHELBY C., 128 Pearl St., Cambridge, Ma. 02139

MILLMAN, MRS. WILLIAM B., Dir., Rider Col., Trenton, N. J. 08602

MILLS, DR. DOROTHY HURST, Dept. of For. Lang., Chapman Col. Lang. Lab., Orange, Ca. 92666

MIRABEAU, ROCH L., Prof. and Ch., For. Lang., Southern Unir., Baton Rouce, La. 70813

MIREL, BERNARD, Dir. LL, Purdue-Calumet, 6738 Magoun, Hammond, In. 46324

MITROVICH, MIRCo M., Phd, Asso. Prof., Sunrise Acres Rt. 1, New Concord, Oh. 43762

MITTAC, MARLIN M., 710 Garfield Ave., Aurora, Il. 60506

MOEN, MARY E., Instr., Anoka Ramsey State Jr. Col., 7154 NE Riverview Terr., Minneapolis, Mn. 55432

MOI, PROF. GEORGE, Camrose Lutheran Col., Camrose Alberta, CANADA

MOISSIY, TAMARA S., Dir. Lang. Lab., Curry Col., Milton, Ma., 10 Mt. Pleasant St., Hyde Park, Ma. 02136

MONGILLO, FRANCES, 179 Huntinghill Ave., Middleton, Cn. 96457

MONSON, EDITH L., Chmn. For. Lang. Dept., Georgetown Col., Georgetown, Ky. 40324

MONTAGUE, COLONEL H. P., 196 Beach 128th St., Belle Harbor, N. Y. 11694 


\section{Membership Directory}

MOORE, DR. J. MICHAEL, Metsa Col., San Diego, Ca. 92111

MOORE, MERRIAM, Dpt. Ch. FL, Ridgefield Hi and E. Ridge Jr. High, P. O. Box 353, Ridgefield Ct. 06877

MORAN, SR. M. CATHERINE, OP, Edgewood Col. Sacred Heart, 855 Woodrow St., Madison, Wi. 53711

MORENO, DR. ANTONIO, Span. Prof., Wash and Jefferson Col., 752 Lockhart St., Washington, $\mathrm{Pa} .15301$

MORGAN, MRS. D. K., Dept. of Romance Langs., Univ. of Alberta, Edmonton 7, Alberta, CANADA

MORGAN, MISS ELISABETH, Mid High School Lib., 103 W. Hagerman, Carlsbad, N. M. 88220

MORGAN, MARGOT, LL Dir., Sacred Heart Dominican Col., 2401 E. Holcombe, Houston, TX. 77021

MORGENROTH, ROBERT L., Hd., Dept. For. Lang., 101 Watson Hall, Dekalb, Il. 60115

MORRISON, ROBERT R., C M L, Southern Missionary Col., P. O. Box 475, Collegedale, Tn. 37315

MORTON, DORIS, Dir. Communications Lab., Col. of Emporia, Emporia, Ks. 66801

MOSER, WORTH S., Chm. Dept. Fl., Darlington School, Rome, Ga. 30161

MOUNTAIN, CLIFF, Dir. F L, Mesa Col., 1120 North Ave., Grand Jct., Co. 81501

MULHOLLAND, CHARLES V., Univ. R. I., Language Lab. Supv., Dept. of Lang., Kingston, R. I. 02881

MUNIZ, GERALDINA ORTIZ, Dept. For. Lang., Eastern Illinois Univ., Charleston, Il. 61920

MURDOCH, CHRISTINE, Instr., Southern Missionary Col., Box 422, Collegedale, Tn. 37315

MURPHY, SR. EVELYN, Col. of Great Falls, Great Falls, Mt. 59401

MURPHY, HAROLD T., A P Sp., Marshall Univ., P. O. Box 238, Huntington, W. V. 25701

MURRAY, ANDREW H., Coord. Lang. Lab., Suffolk Comm. Col., P. O. Box V, Stony Brook, N. Y. 11790

MYERS, M. KEITH, Assoc. Prof., Univ. of Il., 1009 S. Westlawn, Champaign, Il. 61820

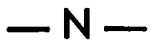

NABERS, JAMES E., Dir. L L, Southern Il. Univ., Dept. of For. Lang., Carbondale, Il. 62903

NACHREINEI, E., Assoc. Prof. of Ger., Andrews Univ., Berrien Springs, Mi. 49104

NAJERA, MRS. VALESKA S., ISLL, Northeastern Il. St. Col., 401 Berkley, Elmhurst, Il. 60126

NARVAEZ, LEON ORLAN, Tchng., U. of Mn. Asst., LL, 2030 Brewster St., Apt. 13, St. Paul, Mn. 55108

NASH, DONALD S., Roberts Wesleyan Col., North Chili, N. Y. 14514

NAVAL ACADEMY, U. S., Modern Languages, Annapolis, Md. 21402

NEHER, WILLIAM R., Hd., Lang. Dept., Breck School, 4200 W. River Rd., Minneapolis, Mn. 55406

NELSON, MARY H., 1800 Clarke Dr., Dubuque, Ia. 52001

NEMNI, MRS. MONIQUE, Dir. L P, Glendon Col., Fr. Dept., 2275 Bayview Ave., Toronto 12, Ontario, CANADA

NESHER, ROBERT A., 425 S. Westend Ave., Lancaster, Pa. 17603

NEVADA, UNIV. OF, Dept. of For. Lang. and Lit., Reno, Nv. 89507

NEWMAN, BERNARD, Tchr. in Ch. Lang. Lab., Bayside High, 32-24 Corp. Kennedy St., Bayside, N. Y. 11361

NEWMAN, JAMES L. V., Simmons Col., 300 The Fenway, Boston, Ma. 02115

NEWMAN, RICHARD W., Chmn., For. Lang. Dept., 625 Huntington Ave., Boston, Ma. 02125 


\section{Membership Directory}

NEWMARK, LEONARD, Chmn., Dept. of Ling., Univ. of Ca., La Jolla, Ca. 92037 NICHOLAS, ST., SCHOOL, 1501 10th, Seattle, Wa. 98102

NICHOLSON, CHARLES E., Chmn., Mod. For. Lang., Ks., St. Dept. Pub. Instr., 120 E. 10th St., Topeka, Ks. 66612

NIXON, RUTH A., Chmn. FL, Wi. State Univ., La Crosse, Wi. 54601

NOEL, SR. M., Instr., Felician Col., So. Main St., Lodi, N. J. 07644

NOMADY, MRS. JANICE, Chmn., Fr. Dept., Colby High School, Colby, Wi. 54421

NORTH TEXAS STATE UNIV., Library, Box 5188,N T Sta., Denton, Tx. 76203

MORTON, DOROTHY M., 1960 Eden Park Blvd., McKeesport, Pa. $15^{132}$

NORWICH, THEODORA, Lang. Lab. Dir., Western College for WMN, Oxford, Oh. 45056

NOVAK, PROF. RICHARD, Dir. Lang. Lab., Concordia Col., 171 White Plains Rd., Bronxville, N. Y. 10708

NOVAK, WITOLD, LL Dir., Audio-Visual Ctr., Monmouth Col., Monmouth, Il. 61462

NUFFER, J. STANLEY, FL Lab., Portland State Col., P. O. Box 751, Portland, Or. 97207

NUUN, ROBERT R., Dir., Bowdoin Col., Brunswick, Me. 04011

NYE, MRS. JEAN, Assoc. Prof. M. L., Findlay Col., Findlay, Oh. 45840

OBRATH, KARL W., Inst. LLD, Univ. of Cincinnati, 700 Riddle Rd., No. 202, Cincinnati, Oh. 45220

O'DELL, DAVID T., Hd. Lang. Dept., Purnell School, Pottersville, N. J. 07979

OJADA, AMPARO B., L L Dept., Loyola Univ., N. Sheridan Rd., Chicago, Il. 60626

OKLA. BAPTIST UNIV., Mod. Lang. Lab., Dir., Shawnee, Ok. 74801

OKSENHALT, DR. SVEIN, Head, Dept. of For. Langs., Eastern Montana Col., Billings, Mt. 59101

OLIVER, DAVID M., 8900 S. Chappel, Chicago, Il. 60617

OLSEN, IRVING S., Dir. Instr. Mats., Val Paraiso Univ., Mod. For. Lang. Rm. 147 Valparaiso, In. 46383

O'NEIL, WILLIAM J. O. JR., Ch/Techn., N. Y. Univ., 64-85 84th St., Rego Park, N. Y. 11379

OP, SR. MARY JEAN, Ch. FLD, Dir. Lang. Lab., Barry Col., Miami, Fla. 33161

OTTO, DR. VIRGINIA, Chmn. Fl. Dept., Nazareth Col. of Rochester, 4245 East Ave., Rochester, N. Y. 14610

OWENS, ROBERT, Chmn. Lang. Dept., Univ. of S. Al., Mobile, Al. 36608

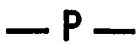

PADEN, TERRENCE, Dir. of Lang. Lab., Upper Ia. Col., POB 583, Fayette, Ia. 52142

PAQUETTE, ClAUDE A., Lang. Lab. Dir., For. Lang. Dept., Univ. of Bridgeport, Bridgeport, Ct. $0 \$ 3602$

PAQUETTE, F. A., Exec. Sec., ACTFL, 62, Fifth Ave., New York, N. Y. 10011

PARHAM, JOSEPH, Asst. Prof., Lang. Lab. Dir., West Georgia Col., Carrollton, Ga. 30117

PARIS, B. E., ASSO. Prof., Faculty of Educ., Sec. Dept. Regina Campus, Regina, Sask., CA'TADA

PARSONS, MRS. ELAINE, Lang. Lab., Green Mountain Col., Poultney, Vt. 05764

PARTINO, ARMANDO, Defense Lang. Instr., E C Br., US Naval Sta., Navy Annex, Annacostia Wa., D. C. 20390

PAST, DR. RAY. Dept. of Eng., The Univ. of Tx., El Paso, Tx. 79999

PATRON, GLORIA A., Dir. L L. La. St. Univ., Lake Front. New Orleans, La. 70122

PAUCHET, JACQUELINE, L L Dir., Knoxville, Col., Rt. 4, Ruggles Ferry Rd., Knoxville, Tn. 37914

PAUCKE, E., Dalton Hall, Bryn Mawr Col., Bryn Mawr, Pa. 19010 


\section{Membership Directory}

PAUL, CHRISTA E., L L Dir., Pa. Col. of Pharm. and Sci., 43rd St. and Kingsessing Ave., Philadelphia, $\mathrm{Pa} .19147$

PAYNE, EDWARD W., P E, Sup. LL, Malone Col., 515 25th St. NW, Canton, Oh. 44709

PAZERIC, ANASTAS, Fr. Tchr., Adams High School, Rochester, Mi. 48063

PAZIK, PROF. RONALD S., Assoc. Prof., Univ. of Windsor, Windsor, Ontario, CANADA

PEAK, J. HUNTER, Hd. Prof., Auburn Univ., FLD, Mell Hall 205, Auburn, Al. 36830

PEARSON, ROSE D., Supv. Lang. Lab., Fairfield Univ., 1540 Unquowa Rd., Fairfield, Ct. 06430

PELOQUIN, PAUL D., Chicopee Comp. High School, Rolf Ave., Chicopee Fallsy, Ma. 01020

PENCHOEN, THOMAS, Ap O BL, Boyce Hall 302, UCLA, 405 Hilgard Ave., Los Angeles, Ca. 90024

PENSACOLA JR. COL., Dir. Lang. Lab., 100 Col. Blvd., Pensacola, Fl. 32504

PENTA, RICHARD M., Coord. of For. Lang., 38 Normandy Rd., Lexington, Ma. 02173

PEREZ, J., Sabido, Lansing Comm. Col., 4106 Windward Dr., Lansing, Mi. 48910

PEREZ, MARIO F., Lang. Lab. Dir., Brevard Col., Brevard, N. C. 28712

PERRY, LCDR F. A., Quarters 4202-H, USAF Academy, Co. 80840

PETERSEN, DR. PHILIP, Dept. of Span. and Port., Stanford Univ., Stanford, Ca. 94305

PETERSON, PAUL W., Div. Dept. Mod. Lang., Gannon Col., Erie, Pa. 16501

PETHERBRIDGE, D. L., Assoc. Prof., Univ. of Lethbridge, Lethbridge Alb., CANADA

PETIT, B. F., Newfield High School, Centereach L. I., N. Y. 11720

PETRIE, REV. R., A-V Coord., St. Joseph Seminary, Callicoon, N. Y. 12723

PETRIZZI, DR. DANIEL J., Coord. FL, Eisenhower Col., Seneca Falls, N. Y. 13148

PETRU, MIRCEA, SC Asst., Kingsborough Comm. Col., Box 700, Brooklyn, N. Y. 11235

PETTIT, JOHN A., Head, M. L. Dept., Marietta Col., Marietto, Oh. 45750

PETTY, JANE P., A Prof. Ger., Bakersfield, Col., LL Cord., 1801 Panorama Dr., Bakersfield, Ca. 93305

PEYRAZAT, J. E., Lang. Lab. Dir., Roanoke Col., Salem, Va. 24135

PHILIPPON, CHRISTIAN, Univ. of Cal-Lang. Lab., Hostal Horche-Gaztambide. 59 Madrid 2, Spain

PHILLIPS, STANLEY E., AV Supv., Boyce Campus Comm. Col., Mosside Blvd., Monroeville, Pa. 15146

PHYLIIS, SISTER M., Notre Dame Preparatory, 815 Hampton Lane, Baltimore, Md. 21204

PICCO, MRS. ELSIE V., Lab. Supv., Bennington Col., Bennington, Vt. 05201

PILRANIS, WILlIAM J., Lang. Lab Dir., R.. I. Jr. Col., 199 Promenade St., Providence, R. I. 02908

PIMSLEUR, DR. PAUL, Dir/List. Ctr., Ohio State Univ., 164 W. 17th Ave., Columbus, Oh. 43210

PIOLA, GLADYS, Lang. Lab. Dir., New Canaan High School, New Canaan, Ct. 06840

PIPHER, C. E., Mgr. Br. Oper., Ampex Chicago, 5422 W. Touhy Ave., Skokie, Il. 60076

PLASTRE, M. GUY, Faculte Des Lettres, Universite Laval, Quebec, Canada

PLAYOUST, REV. ALFRED G., SJ, Xavier School, P. O. BoX 657, Manila Philippines

PLEASANT, PROF. J. V., Dir. Fr., Columbia Univ., Philosophy Hall, New York, N. Y. 10027

POINZ, D. L., Lang. Lab., Ellis Hall, Ohio Univ., Athens, Ohio 45701 


\section{Membership Directory}

POIRIER, DR. ROGER A., Lab. Dir., Union Col., Springfield Ave., Crawford, N. J. 07016

POISSANT, HERVEY, St. Francis Col., Pool Rd., Riddeford, Me. 04005

POND, SR. M. LELIA, Dir. L. L., Univ. of Dallas, Univ. of Dallas Sta., Dallas, Tx. 75061

PORTERA, JOHN, Lab. Dir., San Fernando Valley State Col., 1811 Nordhoff St., Northridge, Ca. 91324

POWELL, MRS. RICHARD, Lang. Lab., New Canaan High School, New Canaan, Ct. 06840

POWERS, JAMES R., Sr. Supv., Ma. Dept. of Ed/Mod F L, 182 Tremont St., Boston, Ma. 02111

POWERS, JAMES R., Dir. Lang., Cambridge Public Schools, 1700 Cambridge St., Cambridge, Ma. 02138

PRADES, B. J., Sp. Instr. and Dir., Dir. Lang. Lab., 444 Armstrong Hall, W. Va. Univ., Morgantown, W. V. 26506

PRATT, JUDITH, Chrmn., Oswego High School, West First St., Oswego, N. Y. 13126

PRESTON, PROF. F. L., Assoc. Prof., Denison Univ., Box 267, Grandville, Oh. 43023

PRINCIPIA, THE, 13201 Clayton Dr., St. Louis, Mo. 63131

PROCOPIO, DOMENIC R., Chmn., Dept. of For. Lang., Lowell State Col., Lowell, Ma. 01854

PROTASE, SR. M., Prof. Fr., Col. of St. Francis, 500 Wilcox St., Joliet, Il. 60435

PUBL. PROCUREMENT CTR., P. O. Box 9355, Arlington, Va. 22209

DIR.-ENGLISH DEP., Fac. of Gen. Studies, Univ. of Puerto Rico, Rio Piedras, P. R. 00931

PUPO, MARIE-LOUISE, 29-31 N. Poplar St., Mt. Carmel, Pa. 17851

PURICELLI, SR. AGNES, CSJ, Fontbonne Col., Instr., 6800 Wydown Blvd., St. Louis, Mo. 63105

PUSCHENDORF, GUNTHER F., Sup. Lab., Univ. of Ca. Berk., 75 Menlo Ave., W. Lake Daly City, Ca. 95015

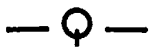

QUEYJA, MICHAEL T., Dir. ML Lab., Univ. of Mis.,University, Mis. 38677

QUINN, T., Grad. Stu., Ohio State Univ., 1384 Jones Tower, Columbus, Oh. 43210

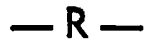

RABKE, MRS. BLANCH, Fr. Tea., John Marshall H. S., Rite 17 Box 48, San Antonio, Tx. 78228

RACINE, DANIEL L., Fr. Instr., Howard Univ., Dept. of Romance Lang., Washington, D. C. 20009

RADTKE, J., Head, Mod. Lang., Marquette High School, 3401 W. Wi. Ave., Milwaukee, Wi. 53208

RADUNZ; HERMAN G., Lang. Lab. Asst., Vancouver City Col., I-3548 W. 4th Ave., Vancouver 8, BC, CANADA

RAGLE, GORDON, A Prof. Span., Rockhurst Col., 5225 Troost Ave., Kansas City, Mo. 64110

RAICHLE, LOUIS P., Prof., Staten Island Comm. Col,, Ocean Terrace, Staten Island, N. Y. 10301

REDDEN, JAMES E., A P of Eng., Southern Illinois Univ., Department of Eng., Carbondale, Il. 62901

REESE, L. GRANT, Chmn., Dept. of Mod. Lang., Memphis State Univ., Memphis, Tn. 38111

REESE, MR. WINSTON J., LL Dir., Univ. of Iowa, 125 Schaeffer Hall, Iowa City, Ia. 52240 


\section{Membership Directory}

REGAN, TIMOTHY F., VP, educational systems corp., 1211 Conn. Ave. NW-S No. 301, Washington, D. C. 20036

REICHARD, JOSEPH R., Dept. of Ger. and Rus., Oberlin Col., Oberlin, Oh. 44074 REID, CONSTANCE L., Eigenmann Ctr., Indiana Univ., Bloomington, In. 47405 REID, MRS. FRANCES, Supv., Lang. Lab., Davidson Col., Davidson, N. C. 28036 REINER, COL HOWARD, 37 Walnut Ave., Wheeling, W. V. 26003

RICHARDSON, CHARLES P., DLL, Ellis Hall, Ohio University, Athens, Ohio 45701

RICHARDSON, SISTER JANET, Chmn. FL Dept., Englewood Cliffs Col., Englewood N. J. 07632

RIEP, A. R., Chmn., Dept. of Mod. Lang., Wartburg Col., Waverly, Iowa 50677

REITINGER, DR. GOTTFRIED, Universtat Innsbruck, Fischnalerstrase 4, 6020 Innsbruck, Austria

REMEIKA, MRS. RUTH N., 79 Quabeck Ave., Irvington, N. J. 07111

RETAILLIAU, SIMONE, Fordham Univ., 2940 Grand Concourse, New York, N. Y. 10458

RHODE, SR. ELIZABETH, Ch. LD, Alverno Col., 3401 South 39 St., Milwaukee, Wi. 53215

RIUSECH, ALBERT, A Prof. of Lang., Ouachita Baptist U., Box 739, Arkadelphia, Ar. 71923

RIVERS, DR. W. M., Box 66, Tea. Col., Columbia Univ., 525 W. 120 St., New York N. Y. 10027

ROBERTSON, DR. GEORGE T., Prof. of Fr., Eastern Wash. St. Col., Cheney, Wa. 99004

ROBILLARD, MRS. MADELEINE T., Amherst Senior Hg., 219 Audubon Dr., Snyder, N. Y. 14226

ROBINSON, CLAUDETTE, 2135 Gilford, Montreal, Quebec, CANADA

ROBINSON, FRANCES, Hd., Lang. Dept., Newington High, 131 Cedar St., Newington, Ct. 06111

RODGERS, MARY LUNN, Chmn., Dept. For. Langs., Box 643 H-S Sta., Abilene, Tx. 79601

RODRIGUEZ, MRE. BERTA, Dir. of Lang. Lab., Oxford Col., Emory Univ., Oxford, Ga. 30257

ROEMING, ROBERT F., Dir., CTR The Cent. Studies, Univ. Wi.-Mil., Milwaukee, Wi. 53201

ROHRER, MRS. G. W., Ch. F. L., 2712 Bradford Dr., Toledo, Oh. 43614

ROLFE, DR. O. W., Asst. Prof. Romance Lang., Univ. of Wa., Seattle, Washington 98105

ROSARIO, SR., Chmn. Dept. FL, Col. of the Holy Names, 3500 Mountain Blvd., Oakland, Ca. 94619

ROSARIA, SR. MARY, CSSF, Good Counsel H. S., 3900 Peterson Ave., Chicago, Il. 60645

ROSELDA, S. M., Head Mod. Lang. Dept., Mater Dei .High School, 9th and Plum St., Breese, Il. 62230

ROSENDA, SR., DML, Lang. Lab. Coord., Regis Col., Weston, Ma. 02193

ROSIC, PROF. M., Dept. of Rus., Knox Col., Galesburg, Il. 61401

ROSS, CHARLES E., Tchng. Ast., Univ. of Southern Calif., 10711 Rose Ave., Los Angeles, Ca. 90034

ROVNER, DR. PHILIP, Univ. of Maryland, Dept. of Span. and Port., College Park, Md. 20742

ROY, PROF. J., Dept. Fr., Rm. A351 Arts Bldg., Lauren Ramsey Lake/Tian Univ., Sudbury, Ontario, Canda

RUBIN, KATHLEEN KAY, Regis Col., W. 50th Ave., and Lowell Blvd., Denver, Co. 80221

RUGGLeS, GORDON W., Dir., Ashland Col., Col. Ave., Ashland, Oh. 44805

RUMPF, MARCELLE, Lect., Univ. of Lethbridge, Lethbridge Alberta Canada 


\section{Membership Directory}

RUPPRECHT, REV. MELVIN, Lab. Dir., St. Vincent Col.. Latrobe, Pa. 15650

RUSCH, NORMA, Lang. Lab. Dir., Lakeland Union High Sch, Minocqua, Wi. 54548 RUTE, MRS. MARY, Tape Libr., Harvard U., Mod. Lang. Ctr., Boylston Hall, Harvard Yd., Cambridge, Ma. 02138

RYAN, MRS. CAROLE ANN, Asst. Prof. of Fr., Illinois Col., Jacksonville, Il. 62650 RYBERG, LILLIAN, Bethel Col., 1480 N. Snelling, St. Paul, Mn. 55101

\section{$-\mathrm{S}-$}

SACHER, CECIL, Chr. FL, Niles Twp. HS N., 9800 Lawler, Skokie, Il. 60076

SADBERRY, DR. LONNIE, Prof. of Span., Prairie View A \& M Col., Prairie View, Tx. 77445

SALAS, SAMUEL A., Dir. L L, Cranbrook School, 520 Lone Pine Rd., Bloomfield Hills, Mi. 48013

SALERNO, DR. GRAYCE, C D FL, Dept. Eng. and For. Lang., Bergen Community Col., Paramus, N. J. 07652

SAMANIEGO, F. A., Instr., Utah State Univ., Dept. of Lang., Lagan, Ut. 84321

SAMPON, VICTOR, CH FL, Lang. Lab. Dir, Wayland Aca., Beaver Dam, Wi. 53916

SANCHEZ, MRS. MARY, Supv., For. Lang., P. O. Box 4688, Clearwater, F1. 33518

SANDSTOM, ELEANOR, Dir. Lang. Labs., School Dist. of Pa., Parkway and 21st. Philadelphia, Pa. 19103

SANTOMO, BENIGNO F., Esl. Tea., P. O. 1134, Redondo Bch., Ca. 90278

SAUNDERS, HELEN V., W. Va. State Dept. of Edu., 1716 Franklin Ave., Charleston, W. V. 25305

SAWYER, J. O., Dir. Lang. Lab., Univ. of Ca., Berkeley, Ca. 94720

SCARTH, MARGARET, Asst. Prof. in Span., Erindale Col., U. Toronto, Toronto 5 , Ontario, CANADA

SCHABACKER, MISS RUTH, 129 Kent Place Blvd., Summit, N. J. 07901

SCHAEFER, U. M., Supv., Lang. Lab., Connecticut Col., Box 1524, New London, Ct. 06320

SCHAUBACHER, ANNE MARIE, Chmn., French Dept., Shipley School, Bryn Mawr, Pa. 19010

SCHEFSKY, CARL W.. Inst. Med., Univ. of Portland, $5000 \mathrm{~N}$. Willamette Blvd., Portland, Or. 97203

SCHMIDT, HANS, 51 Mannakee St., Rockville, Md. 20850

SCHMITZ, VICTORIA, Assoc. Dir., Schoonover Lab., Ohio Northern Univ., Ada, Oh. 45810

SCHMOLL, RONALD B., Asst. Prof. of Ger., Asheville-Biltmore Col., Asheville, N. C. 28801

SCHOBEL, MRS. DIANNE, 7801 Sagamore Dr., Cincinnati, Oh. 45236

SCHOLTIS, EBERHARD, Lang. Lab. Dir., Plattsburgh State U. Col., Plattsburgh, N. Y. 12901

SCHORMULLER, MRS. M. N., P. O. Box 405, Painesville, Oh. 44077

SCHULTZ, DAVID, Lehman College Cuny, Bedford Park Blvd., Bronx, N. Y. 10468

SCHULTZ, GEORGE F., A. Prof. of ML, Central State Univ., 109 N. Washington St., Columbia City, In. 46725

SCHWANK, DENNIS M., Lang. Lab. Coord., University of Mn., Morris, Mn. 56267 SCHWENKER, D., LL Mgr., Univ. of Guelph, 230 Edinburgh Rd., S., Guelph, Ont., CANADA

SCOTT, S. N., Instr. Ling., Kirkland Col., Clinton, N. Y. 13323

SCULLY, MRS. P., R. P. 1. 285 Victoria St., Toronto, Ontario, CANADA

SEARCH, RUSSEL H., Dir. AV Materials, Bucknell Univ., Lewisburg. Pa. 17837

SEATTLE COMMUNITY COL. SYS., Mod. Lang. Dept., Washington Community Col., Dist. VI, 1718 Broadway, Seattle, Wa. 98122

SECONDARY TEACHERS COL., The Librarian, Private Bag, Auckland, N. Zealand 


\section{Membership Directory}

SERAFINO, ROBERT P., Supv. of For. Lang., New Haven Pub. Sch., P. O. Box 3816, New Haven, Ct. 06525

SENG, MARK W., Ass't. Prof., Univ. of Tx., Austin, 2505 Princeton Dr., Austin, TX. 78741

SERRANO, CARLOS, Stu., 75 Alexandra St., Waterloo Ontario, CANADA

SEXTON, MRS. EILEEN, Bronx Comm. Col., 120 E. 184 St., Bronx, N. Y. 10468

SHAKE, EDWIN D., L L Dir., Stephen F. Austin St., Col., Nacogdoches, Tx. 75961

SHAPIRO, MRS. MARY, State Col., Bridgewater, Ma. 02324

SHARPE, P. B., Dean Jr. Col., 132 Main St., Franklin, Ma. 02038

SHAW, DR. ARACELIS G., Romance Lang., Columbia Col.. Columbia, S. C. 29203

SHEARER, JULIE, Asst. Prof., Central Wesleyan Col., Edgewood Arms No. 4, Clemson, S. C. 29631

SHEEHAN, JOSEPH H., Georgetown Univ., Washington, D. C. 20007

SHEEHAU, PROF. JOSEPH, Extfp. Suite 300,3600 M. St. NW, Washington, D. C. 20007

SHeETS, WALTeR A., F. L. Supv., Bartholomew Cons. Sch. Corp., 1400 25th St., Columbus, In. 47201

SHERBAN, DIANE, Tea., Gilmour Aca., Cedar and Son Center Rds., Gates Mill, Oh. 44040

SHERBURNE, ROBERT, Cazenovia Central School, 2 Evergreen Ln., Cazenovia, N. Y. 13035

SHERRARD, DAVID A., Chief/AV Br., DLIEC, US Naval Sta., Anacostia An., Washington, D. C. 20390

SHIELDS, MARION D., MFL Dept. Head, Dunedin High School, Dunedin, Fla. 33528

SHIREY, MARY M., 2419 Longview Ave., SW, Roanoke, Va. 24014

SHORES, LOREN W., Ch. For. Lang., Scarsdale H. S., Scarsdale, N. Y. 10583

SHREFFLER, E. H., L L Dir., Austin Col., Box 1239, Sherman, Tx. 75091

SIMMONS, DOUGLAS J., Asst. Pr., Dept. of For. Lang., Arizona St. Univ., Temple, Az. 85281

SIMMONS, HOWARD L., Lab. Dir., Forest Park Comm. Col., 38 Plaza South, Apt. 705 , St. Louis, Mo. 63103

SIMMONS, S. J. JOSEPH, Head, Georgetown Prep. Sch. M L, 1225 Otis St., NE, Washington, D. C. 20017

SIREVAAG, JOHN, Head. Ger. Dept., Augustana Col., Rock Island, Il. 61202

SENECAL, JEAN PAUL, 70-3 D Auvergne, Longueuil PQ, CANADA

SKAHSE. PETER. Dir. L L, Wheaton Col., 425 E. Oak Ave., Wheaton, Il. 60187

SKWAREK, RUTH, Instr., Muskegon City Comm. Col., 15088 W. Leonard Rd., Spring Lake, Mi. 49456

SMALL, SOL, Technical Dir. Lang. Lab., C. W. Post Col., Brookville, N. Y. 11548

SMITH, DONALD C., 4035 Burrmont Rd., Rockford, Il. 61107

SMITH, FRANCIS T., Dir. Lang. Lab., Seton Hall U., So. Orange, N. J. 07079

SMITH, WM. FLINT, Dir. L. L., Purdue Univ., Dept. of Mod. Lang., Lafayette, In. 47907

SMITH, DR. PHILIP D., P C, West Chester St. Col., High and Rosedale Ave., W. Chester, $\mathrm{Pa} .19380$

SMITHER, WILLIAM J., Prof. Sp., Tulane Univ., Dir. Lang. Lab., New Orlears, La. 70118

SNEAD, EDWARD, Coord. For. Lang., Grambling Col., P. O. Box 235, Grambling, La. 71245

SNYDER, SR. JANE DE CHANTAL, Fontboone Col., FL Dept. Dir., 6800 Wydown Blvd., St. Louis, Mo. 63105

SODERMAN, LEONARDO, Lang. Lab. Dir., Univ. of Concepcion, Casilla 2307 Concept, CHILE

SOKALSKI, ALEX, Univ. of Bridgeport, Dept. of FL, Bridgeport, Ct. 06602

SOKOLOW, HENRY N., Dir. Lar.g. Labs., Santa Monica City Col., Santa Monica, Ca. 90401 


\section{Membership Directory}

SPEAR, FREDERICK A., Assoc. Prof. of Fr., Skidmore Col., Saratoga Spgs., N. Y. 12865

SPECTOR, NORMAN B., Chmn., Dept. Fr. and Ital., Northwestern Univ., Evanston, Il. 60201

SONOMA STATE COL., Receiving Dept., 1801 E. Cotati Ave., Rohnert Park, Ca. 94928

SOULE, DENNIS, Instr., Bethany Lutheran Jr. Col., Mankato, Mn. 56001

SPENCE, PATRICK, Asst. Dir., Baptist Col., P.- O. Box 10087, Charleston, S. C. 29411

SPETRINO, ANTHONY, Ch. FLD, Bassick High School, 1181 Fairfield Ave., Bridgeport, Ct. 06605

SPINKS, O. R., Dir., Suny. At Albany, Humanities B-16, Albany, N. Y. 12203

STACK, E. M., Prof., N. C. State Univ., 3925 Arrow Dr., Raleigh, N. C. 27609

STADT, MRS. B. W., Sp. Prof., Rollins Col., Box 119, Winter Park, Fl. 32789

STEFFEN, ROBERT, Sup. Syracuse Univ., Record Dept., 240 H. B. Crouse Bldg., Syracuse, N. Y. 13210

STEINBERG, ADRIAN L., Dir. F. L. L., Temple Univ., 3rd. Floor, Col. Hall, Philadelphia, Pa. 19122

STERN, RHODA, 305 E. 24th St. 18B, New York, N. Y. 10010

STEVENS, EDITH, Methods Ins., Moorhead State Col., 1211 4th Ave. S., Moorhead, Mn. 56560

STEWART, ANDREW T., Hd., For. Lang. Dept., Cardigan Mountain School, Canaan, N. H. 03741

STEWART, MRS. DORIS L., 4823 S. Land Park Dr., Sacramento, Ca. 95822

STEWART, HARRY E., 600 South Col., Tulsa, Ok. 74104

STITES, KENNETH, Dir. Lang. Lab., UNC-W, Wilmington, N. C. 28401

STONER, MARJORIE M., Ex. Sec., Southwestern U. at Memphis, Lang. Ctr., Memphis, Tn. 38112

SUAREZ, J. DAVID, Inst. Mod. Lang., State Col. at Boston, 19 Blackthorn Rd., Framingham, Ma. 01701

SULLIVAN, MR. JAMES, 4914 Main St., Lisle, Il. 60532

SULLIVAN, JAMES J., Pres., James J. Sullivan Corp., 1517 Ogden Ave., Downers Grove, Il. 60515

SUMMIT LAB DIR., Board of Educ., 97 Maple St., Summit, N. J. 07901

SUTTON, LOIS M.. Prof. of Fr., Baylor Univ., Waco, Tx. 70703

SWANSON, MR. SEVERIN A., RR 1, Box 135, Omro, Wi. 54963

SWETT, DR. DOUGLAS, Chmn. Dept. For. Lang., Princopia Col., Elsah, Il. 62028

SWIFT, MRS. MARTHA H., Morgan Park Aca., 2153 West 111th St., Chicago, Il. 60643

SYGODA, Ivan R., 133 Pa. Ave., Oxford, Pa. 19363

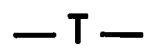

TADEY, MISS B., Hd. Lang. Dept., Gibault High School, 501 Columbia Ave., Waterloo, Il. 62298

TAGGART, F., Sp. Tea., Ojai Valley School, El Paseo Rd., Ojai, Ca. 93023

TAGGART, GILBERT, Prof., Sir Geo. Williams Univ., 1435 Drummend St., Montreal 107 PQ., CANADA

TANNER, MRS. J. M., Dir. Supv., Univ. of Virginia, 302 Cabell Hall, Charlottesvile, Va. 22903

TARAS, DR. A. F., Dir. Audio Lab Ctr., Ithaca Col., Ithaca, N. Y. 14850

TAYLOR. WALTER C., Dept. of Span., Upsala Col., E. Orange, N. J. 07019

TEXAS STATE UNIV, N., Acquisitions Libr., Box 5188, N T Sta., Denton, Tx. 76201

THADDEUS, SISTER JUDE, Chmn. Span. Dept., Seton Hill Col., Greensburg, Pa. 15601 


\section{Membership Directory}

THARAUD, MRS. CYNTHIA, Lang. Lab. Dir., 308 Philosophy Hall, Columbia Univ., New York, N. Y. 11027

THATCHER, J., Ast. Hd/Mod. LD, Burlington Central Hi. Sch., Box 5042, Burlington, Ontario, CANADA

THERESE, SR. MARIE, Chmn. FL Dept., Good Counsel Col., 52 N. Broadway, White Plains, N. Y. 10503

THERRIEN, MARCELLA, Lang. Lab. Dir., 117 Margern Rd., Welland, Ontario, CANADA

THEUMA, MR. JEAN R., Dir. FL, Univ. of Hawaii, 2528 The Mall, Rm. 303, Honolulu, Hawaii 96822

THIBAULT, CLAUDE, Ecole Normale N-D-De Foy, Cap-Rouge PQ, Canada

THOM, J. M., Library, E. L. Vandermeulen HS, Old Post Rd., Pt. Jefferson, N. Y. 11777

THOMANN, DONALDO J., Pacific Union Col., Angwin, Ca. 94508

THOMAS, FREDERICK M., Carlson High School, 30550 W. Jefferson Ave., Gibraltar, Mi. 48173

THOMAS, HALEY F., Inst., P. O. Box 403, Univ. of Richmond, Richmond, Va. 23173

THOMAS, JOSEPH V., Prof. of Mod. Lang., Austin Peay State Univ., Clarksville, Tn. 37040

THOMPSON, MRS. C. S., 1820 Cascade St., Fayetteville, N. C. 28301

THOMPSON, GAIL, Lang. Lab., North Central Col., Rall Hall, Naperville, Il. 60540

THORNDIKE, E. KAY, 7938 Military Ave., Omaha, Neb. 68134

THORNE, E. F., Assoc. Prof., West. Liberty State Col., West Liberty, W. V. 26074

THORNHILL, DONALD E., Dir. For. Lang., Westport Bd. Educ., Westport, Ct. 06880

THURMAN, MRS. GARY R., Instr. In Mod. Langs., Huntington Col., Huntington, In. 46750

TOCONITA, DR. M. J., Prof. and Dir. Lang. Lab., St. Joseph S. Col., Philadelphia, Pa. 19131

TODD, THOMAS C., Dir., MATL, School For. Intnl. Training, Kipling Rd., Brattleboro, Vt. 05301

TIRADO, DR. M., Chmn. For. Lang. Dept., Wagner Col., Staten Island, N. Y. 10301

TOIRAC, DOROTHY M., Chmn. Lang. Dept., Grace College, Winono Lake, In. 46590

TOLMAN, JON M., Lect., Indiana Univ., Dept. of Span. and Port., Bloomington, In. 47401

TOMLINSON, M. D., Mod. Lang. Dept., R. 427, Humanities LDG, Univ. of Tn., Martin, Tn. 38237

TORRIELLI, A. J., 820 N. Michigan Ave., Chicago, Il. 60611

TRAHAN, ROGER R., Dir. Lang. Lab., Assumption Col., 500 Salisbury St., Worcester, Ma. 01609

TRIPP, MRS. GERTRUDE M., LLD, Elmhurst Col., 190 Prospect Ave., Elmhurst, Il. 60126

TOVEN, AUDUN, Instr., Pacific Lutheran Univ., Tacoma, Wa. 98447

TULLOS, OSCAR, Texas Southmost Col., 104 Greenway Dr., Brownsville, Tx. 78520

TUMER, MARGUERITE J., Consint., Dayton Bd. of Educ., 354 Bungalow Rd., Dayton, Oh. 45417

TURNER, ALBERT R., Dir. Lang. Lab., 1600 Dodge Ave., Evanston, Il. 60204

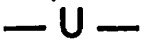

UBBEN, DR. JOHN H., Ch. Dept. Ger.,Labmerton Hall, Lehigh Univ., Bethlehem, $\mathrm{Pa} .18015$

UMMEL, CAROLYN M., Valley View Local Schools, Comstock Ave., Germantown, Oh. $\mathbf{4 5 3 2 7}$ 
VALETTE, REBECCA M., Dir. Lang. Lab., Boston Col., Chestnut Hill, Ma. 02167

VALVERGE, DR. LUIS J., Ch. FLD., Boise State Col., Box 24, Boise, Id. 83701

VANACORE, SR. EVANGELA, Op/Dir., Ohio Dominican Col., 1216 Sunbury Dr., Columbus, Oh. 43219

VANDESTEEG, MARY D., Dir. L. L., Indian Hill Jr.-Sr. H. S. 7370 Shawnee Rd., Apt. 3, Cincinnati, Oh. 45243

VANG, PAUL D., Lang. Lab. Dir., Thiel Col., Greenville, Pa. 16125

VANKERK, JOHN W., Lang. Lab. Dir., Indiana State Univ., 15321/2 N. 7th St., Terre Haute, In. 45705

VAN TREESE, GLENN J., Dir. Lang. Lab., Sweet Briar Col., P. O. Box 18, Sweet Briar, Va. 24595

VAN ZAANAN, DIRK B., Lab. Dir., Rutgers State Univ., 175 Univ. Av-Conklin Hall, Newark, N. J. 07102

VARGAS, EDUARDO E., A Prof., Missouri Western Col., 3402 Bel-Nor Dr., St. Joseph, Mo. 64506

VELEY, ROBERT W., Ch. Dept. FL, Benson Polytechnic Sch., 546 NE 12th Ave., Portland, Or. 97232

VELTMAN, PROF. HUGH E., Grand Valley State Col., 615 Monroe Blvd., S. Haven, Mich. 49090

VERRETTE, VICTOR S., Dir. Lang. Lab., Grinnell Col., Grinnel, Ia. 50112

VESEL, BRO. FRANCIS, St. Michael S. High School Sante Fe, N. M. 87501

VIGIL, NEDDY A., Dir. LL, Univ. of New Mexico, Dept. of Mod. and Clas. Lang., Albuquerque, N. M. 87106

VOGT, M. I., L L Dir., University of Idaho, ad 322 Univ. of Id., Moscow, Id. 83843

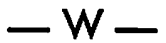

WAGENINGE, MRS. F., 5501 Park Ave., West New York, N. J. 07093

WALK, VAN, GRACE R., Grants Pass High School, 522 NE Olive, Grants Pass, Or. 97526

WALSH, SR. MARIE ANDRE, IHM, St. Edward S. Univ., Dir. LL, 3001 S. Congress, Austin, Tx. 78704

WARDLAW COUNTRY DAY SCHOOL, Language Department, 1030 Central Ave., Plainfield, N. J- 07060

WARE, SALLY LO, Dir. of Lang. Lab., Rockford Col., Rockford, Il. 61101

WARING, THOMAS R. JR., Chmn. Lang. Dept., Sewanee Military Aca., Sewanee, Tn. 37375

WARREN, DR. VIRGIL A., 323 Corprew St., Fayette, Mo. 65248

WASHINGTON, ELEANOR A., Dir./LL, Nettie Lee Roth HS, 727 Oak Leaf Dr., Dayton, Oh. 45408

WASHINGTON UNIVERSITY LIBRARIES, Acquisitions Dept., St. Louis, Mo. 63130

WATKINS, JAMES M., Director, Language Lab., Middlebury College, Sunderland Lang. Ctr., Middlebury, Vt. 05753

WATSON, JACK, Lang. Dir., Williams Col., Karl E. Weston Lang. Ctr., Williamstown, Ma. 01267

WATSON, JOHN A., Dir. For. Lang. Lab., Virginia Union Univ., Richmond, Va. 23220

WATSON, FR. HAROLD, St. Benedict's Col,, Atchison, Ka. 66002

WEBB, DR. HARRY, Audio-Visual Dir., College of St. Thomas, St. Paul, Mn. 55101

WEBBER, DONALD B., Asst. Prof. Lang. Lab., The University of the South, Sewanee, Tn. 37375

WEE, ROBERT, Tea., Ephrata High School 803 Oak Blvd., Ephrata, Pa. 17522

WEINKAUF, DR. ARNOLD L., Ast. Prof. of Lang., Michigan Technological Univ., Houghton, Mi. 49931 


\section{Membership Directory}

WELDING, MRS. GLENDA, Tea. of Fr., 117 E. Madison Ave., Broken Arrow, Ok. 74012

WESTHOFF, JOHN, F L CH, Quincy Junior High, 100 South 14 St., Quincy, Ill. 62301

WHAN, ADELAIDE, LL Dir., Univ. of Southern California, Dept. of Religion, Los Angeles, Ca. 90007

WHEELEY, D. W., Dir. A.V Educ., Brookline High School, Brookline, Ma. 02147 WHEETLEY, DR. W., Dr. AV Education, 194 Boylston St., Brookline, Ma. 02146

WHISNANT, NORMAN, Lab. Dir., Furman Univ., Greenville, S. C. 29613

WHITE ELECTRONIC DEVELOP. CORP., 770 Park Ave., Huntington, N. Y. 11743

WHITE, PAUL S., Raven Ind. Inc., P. O. Box 1007, Sioux Falls, S. D. 57105

WHITE, RICHARD J., Dir. of Lang. Lab., Rich Central High Scho., Olympia Fields, Il. 60461

WHITE, SIDNEY P., Dept. Chmn., Morgan High School, Route 81, Clinton, Ct. 06413

WHITINGTON, G. L., Lang. Lab. Dir., Cooke County Jr. Col., Gainesville, Tx. 76240

WHITMORE, ALLEN P., Ch. Div. of Lang. and Lit., NE Missouri State Col., Kirksville, Mo. 63501

WIDMAIER, KARL, Modern Language Instr., Atlantic Union Col., S. Lancaster, Ma. 01561

WIESE, PETER, Lang. Lab, Dir., Southern Ct. State Col., 501 Crescent St., New Haven, Ct. 06515

NALLD-24

WILEY, RAYMOND A., Assoc. Prof., Lemoyne Col., Syracuse, N. Y. 13214

WILKINS, DR., Dept. Head, For. Langs., Lethbridge Col. Inst., Lethbridge, Alberta, CANADA

WILKINS, DR. GEORGE W. JR., Dir. of Lang. Lab., Tulane Univ., New Orleans, La. 70118

WILKINSON, GENE, Lab. Dir., Kearney State Col., 3612 Avenue E., Kearney, Neb. 58847

WILLEY, MR., Dir. Fr. Dept.. Hyde School, Bath, Me. 04530

WILLECKE, DR. F. H., Ch. For. Lang. Dept., Wagner Col., Staten Isl., N. Y. 10301

WILLIAMS, CHAS. B., Lab. Dir., State Univ. Col., Sheldon Hall, Oswego, N. Y. 13126

WILLIS, DAVID G., Chmn. FL, Christian Bro. Acad., 367 Morris St., Albany, N. Y. 12208

WILLIS, DR. ROBERT, Eng. Dept., 32 Washington Pl., Rm. 32, New York City, N. Y. 10013

WILLIS, MRS. IONE, R. R. 3, Milton, Ky. 40045

WINN, REV. PROF. FRANK M., Eastern Baptist Col., St. Davids, Pa. 19087

WINN, MRS. T., Pearsall High School, Box 1291, Pearsall, Tx. 78061

WINSLOW, MARY, PHD, Prof. of Rom. Lang., St. Mary of Plains Col., Dodge City, Ks. 67801

WIPF, JOE. 874 Bricker Blvd., Columbus, Oh. 43221

WISNIEWSKI, RAY, Dir. Audio Video Services, Grand Valley State Col., Allen. dale, Mi. 49401

WOHR, DAVID L., 1831 N. Bruad St., Philadelphia, Pa. 19122

WOJTKOWSKI, IRENA, Youngstown State Univ., Youngstown, Oh. 44503

WOLFRAM, MillaRD, Curric. Coord., Central Admin. Bldg., 10025 Penn Ave., So., Bloomington, Mn. 55431

WOODFORD, CHARLES H., Harper and Row Publishing Inc., 49 East 33rd. St., New York, N. Y. 10016

WOOLSEY, JAY L., Ger. Instr., Hebron Aca., Hebron, Me. 04238

WOOSTER, THE COLLEGE OF, Dir. Lang. Lab., Wooster, Oh. 44691

WORTHINGTON, MR. M. L., Virginia State Col., Box 428, Petersburg, Va. 23803 
YALAMOW, DR. N.P, Dir. Lanı, U. Que-Pav Lafontaine, 1301 Sherbrooke St. E., Montreal (PQ) CANADA

YALDEN, MRS. J. M., Lect., St. Pat S. Col., 281 Echo, Carleton Univ-Dept, Sp., Ottawa 1, Ontario, CANADA

YEAKEY, M. L., Dept. Chmn. Holland Hall School, 2640 S. Birmingham Pl., Tulsa, Ok. 74114

YODER, ROBERT, Goshen Col., 1005 South 8th St., Goshen In. 46526

YOSHISHIGE, GEORGE S., Aud. Vis. Cent., Leeward Comm. Col., 860 Fourth St., Pearl City, Hi. 96782

YOUNG, JOHN N. K., Dir., FL Dept., The MacMillian Co., 866 Third Ave., New York, N. Y. 10022

YUMICH, GEORGE S., Balisdell Pub. Co., 275 Wyman St., Waltham, Ma. 02154

YZENBAARD, JAMES, Dir. Lang. Lab., University of Kentucky, School of Letters and Langs., Lexington, Ky. 40506

\section{$-\mathrm{Z}-$}

ZARR, VALOIS A., Chmn. Ger. Dept., East High School, 1135 South 17th E., Salt Lake City, Ut. 84108

ZEA, RUDOLPHO, Dir. LL, Loyola College, 4501 N. Charles St., Baltimore, Md. 21210

ZEITUNGSVERTRIEBSAMT, Satzabteilung, Clara-Zetkin-Strasse, (1004) Berlin, Germany

ZIERER, PROF. ERNESTO, Chmn., Dept. de Idiomas y Linguistica, Apartado 315 , Universidad Nacional de Trujillo, Trujillo, Peru

ZIMMERMAN, DOROTHY S., Dir. L. L., Mount Mary College, Milwaukee, Wis. 53222

ZIMMERMAN, ELLEN, L L Dir., Thornridge High School, 150-00 Cottage Grove, Dolton, Il. 60419

ZIMMERMAN, MRS. MARGARET K., Chmn., Euclid Sr. High School, 711 East 222nd. St., Euclid, Oh. 44123

ZUMBAR, MRS. WILlIAM, Asst. Prof. of Spa., Mount Union Col., Alliance, Oh. 44601

ZUZIC, DR. MARKO, Chmn., Salem Col., 174 Liberty St., Salem, W. V. 26426 